Issued by Sandia National Laboratories, operated for the United States Department of Energy by Sandia Corporation.

NOTICE: This report was prepared as an account of work sponsored by an agency of the United States Government. Neither the United States Government, nor any agency thereof, nor any of their employees, nor any of their contractors, subcontractors, or their employees, make any warranty, express or implied, or assume any legal liability or responsibility for the accuracy, completeness, or usefulness of any information, apparatus, product, or process disclosed, or represent that its use would not infringe privately owned rights. Reference herein to any specific commercial product, process, or service by trade name, trademark, manufacturer, or otherwise, does not necessarily constitute or imply its endorsement, recommendation, or favoring by the United States Government, any agency thereof, or any of their contractors or subcontractors. The views and opinions expressed herein do not necessarily state or reflect those of the United States Government, any agency thereof, or any of their contractors.

Printed in the United States of America. This report has been reproduced directly from the best available copy.

Available to DOE and DOE contractors from

Office of Scientific and Technical Information

P.O. Box 62

Oak Ridge, TN 37831

Prices available from (703) 605-6000

Web site: http://www.ntis.gov/ordering.htm

Available to the public from

National Technical Information Service

U.S. Department of Commerce

5285 Port Royal Rd

Springfield, VA 22161 


\section{DISCLAIMER}

Portions of this document may be illegible in electronic image products. Images are produced from the best available original document. 
SAND99-2808

Unlimited Release

Printed January 2000

\title{
On the Automatic Generation of Plans for Life Cycle Assembly Processes
}

\author{
Terri L. Calton \\ Intelligent Systems and Robotics Center \\ Sandia National Laboratories \\ P.O. Box 5800 \\ Albuquerque, NM 87185-1008
}

\begin{abstract}
Designing products for easy assembly and disassembly during their entire life cycles for purposes including product assembly, product upgrade, product servicing and repair, and product disposal is a process that involves many disciplines. In addition, finding the best solution often involves considering the design as a whole and by considering its intended life cycle. Different goals and manufacturing plan selection criteria, as compared to initial assembly, require re-visiting significant fundamental assumptions and methods that underlie current assembly planning techniques. Previous work in this area has been limited to either academic studies of issues in assembly planning or to applied studies of life cycle assembly processes that give no attention to automatic planning. It is believed that merging these two areas will result in a much greater ability to design for, optimize, and analyze life cycle assembly processes.

The study of assembly planning is at the very heart of manufacturing research facilities and academic engineering institutions; and, in recent years a number of significant advances in the field of assembly planning have been made. These advances have ranged from the development of automated assembly planning systems, such as Sandia's Automated Assembly Analysis System, Archimedes $3.0^{\circ}$, to the startling revolution in microprocessors and computercontrolled production tools such as computer-aided design (CAD), computer-aided manufacturing (CAM), flexible manufacturing systems (FMS), and computer-integrated manufacturing (CIM). These results have kindled considerable interest in the study of algorithms for life cycle related assembly processes and have blossomed into a field of intense interest. The intent of this manuscript is to bring together the fundamental results in this area, so that the unifying principles and underlying concepts of algorithm design may more easily be implemented in practice.
\end{abstract}


Intentionally Left Blank. 


\section{Preface}

Many manufacturing companies today expend more effort on upgrade and disposal projects than on clean-slate design, and this trend is expected to become more prevalent in coming years. However, commercial computer-aided design (CAD) tools are better suited to initial product design than to the product's full life cycle. Computer-aided assembly analysis and planning, optimization, and visualization of life cycle assembly processes based on the product CAD data can help ensure accuracy and the reduce effort expended in planning these processes for existing products, as well as provide design-for-life-cycle analysis for new designs. However, to be truly effective, the computer-aided assembly analysis and planning systems must allow users to express their assembly and disassembly plan selection criteria that apply to their products and production environments during the planning sessions.

This manuscript serves as the technical report required by Sandia National Laboratories Laboratory Directed Research and Development (LDRD) Program for the project entitled "Automatic Planning of Life Cycle Assembly Processes". The LDRD Program financially supported the entire project and this manuscript summarizes the technical achievements made throughout the project's three-year history. The work conducted under the purview of the LDRD focuses on automating analysis and planning techniques for later life cycle assembly processes.

Sandia's Automated Assembly Analysis System, Archimedes $3.0^{\circ}$, was used as the foundation for integrating algorithms to automatically generate assembly and disassembly plans for later life cycle assembly processes. The results produced under the purview of this LDRD, together with the results produced under the purview of two other two-year (FY98 and FY99) LDRDs projects, "Ergonomics in Life Cycle Assembly Processes" and "Analysis of Very Large Assemblies", constitute the features used to upgrade Archimedes $3.0^{\circ}$ to Archimedes 4.0.

Archimedes $3.0^{\oplus}$ was copyrighted in 1998 . The copyright for Archimedes 4.0 is in progress. The fundamental planning concepts underlying Archimedes $3.0^{\circ}$ is the manufacturing plan selection criteria, (constraints) framework. A patent release for this framework, combined with the planning algorithms, is expected to be issued early in the calendar year of 2000 .

The goals of this manuscript are to bring together the fundamental results of the algorithms that automatically generate assembly and disassembly plans for later life cycle assembly processes, which were produced for this LDRD, to provide a totally integrated environment for the designer to create a high-quality, cost-effective, optimal product. To better assist the reader of this manuscript, Chapters 1 and 2 are devoted to the manufacturing constraints framework and planning algorithms of the Archimedes $3.0^{\circ}$. The depth of coverage in these first two chapters 
provides the necessary background for the remaining chapters which focus on life cycle assembly processes: product disassembly, product upgrade, product servicing and repair, product costing and product design.

\section{Acknowledgements}

The material in this manuscript has been derived from numerous resources, ranging from countless numbers of research and applied-oriented publications, conferences and workshops, to the extremely knowledgeable people from within academia and industry. A wealth of information has been gathered and assimilated in support of developing computer algorithms required for the automatic generation of plans for: product assembly, product disassembly, product upgrade, product servicing and repair and product disposal. I would like to extend my appreciation to all the people who helped collect and assimilate the information, especially to Randy Wilson at Kodak for sharing his enthusiasm and wisdom on the art and science of assembly planning very early on in the project.

To Ralph Peters, Russell Brown, and Emily Mitchell, I owe tremendous gratitude to for their computer programming endeavors. Ralph was responsible for implementing a design-for-lifecycle cost analysis module and integrating it into the Archimedes $3.0^{\odot}$ System. Russell was largely responsible for implementing the disassembly planning algorithms and for implementing a prototype design and cost tool for small vehicular robots, separate from Archimedes $3.0^{\odot}$. Emily was responsible for implementing the constraint rules to automate generative process planning capabilities for product upgrade. Without their support, none of this work would have been possible.

A special thanks goes to Sharon Blauwkamp, my administrative assistant, for her never-ending willingness to help me with the day-in and day-out activities of office life.

I am also grateful to Karen Crapse at Cummins Engine Inc., for providing her design and manufacturing expertise that led to several of the implemented life cycle algorithms. 


\section{Contents}

Preface iii

Executive Summary ix

1. Constraint-based Assembly Planning 1

1.1. Background and Motivation 1

1.2. Previous Work 2

1.3. Manufacturing Plan Selection Criteria 3

1.4. Assembly Planning Constraints 3

$\begin{array}{ll}\text { References } & 8\end{array}$

2. The Archimedes $3.0^{\Theta}$ Automated Assembly Analysis

System

2.1. Assembly Planning Approach ' 12

2.2. User Interface 13

2.3. When Planning Fails 15

2.4. Efficiency 16

2.5. Implementation $\quad 17$

$\begin{array}{ll}\text { References } & 17\end{array}$

3. Automatic Planning for Complete Disassembly 19

3.1. Introduction 20

3.2. Previous Work 21

3.3. Geometric Reasoning about Assembly Tools 24

3.4. Disassembly Planning Approach 26

References 28

4. Automatic Planning for Manufacturing Generative Process

Planning $\quad 31$

4.1. Background and Motivation $\quad 32$

4.2. Generative Process Planning Issues 34 
4.3. Re-design 35

4.4. Planning with Subassemblies $\quad 37$

4.5. Conflict Resolution between Generative Plans $\quad 40$

4.6. Conclusions and Future Work 44

5. Automatic Planning for Partial Disassembly 45

5.1. Service-oriented Assembly Planning 46

5.2. An Optimizing Search Algorithm for Assembly Sequences 46

5.3. Experiments 41

6. Automating Cost Estimates for Life Cycle Assembly

Processes $\quad 53$

6.1. Introduction $\quad 54$

6.2. Integrated Design-to-Cost $\quad 55$

6.3. Interactivity and Implementation 56

6.4. Experimentation $\quad 57$

7. Small Smart Machines - A Framework for Automating

Design $\quad 59$

$\begin{array}{ll}\text { 7.1. Motivation } & 60\end{array}$

7.2. Implementation. $\quad 60$

7.3. System Inputs. $\quad 60$

7.4. Optimization Criteria. $\quad 61$

7.5. User Interface $\quad 61$

Figures

1. Figure 2.1. The Archimedes $3.0^{\odot}$ assembly planning system. 13

2. Figure 2.2. Panning dialog showing a partial list of constraints. 14

3. Figure 2.3. Planning dialog showing the instantiation of a REQ SUB_WHOLE constraint. 15

4. Figure 3.1. Defining the placement of a socket wrench for a
REQ TOOL constraint.

5. Figure 4.1. The B61 nose assembly. 33 
6. Figure 4.2. Example of the effects of re-design.

7. Figure 4.3. Demonstration of propagation effects for planning with subassemblies.

8. Figure 4.4. Design modification for Subassembly $T_{2}$.

9. Figure 4.5. Design modification for Subassembly $S_{I}$.

10. Figure 5.1. Example of an assembly from which a specific part is to be removed.

11. Figure 5.2. Example of an assembly where the shortening algorithm is not guaranteed to find an optimally short disassembly sequence.

12. Figure 5.3. The tail-section subassembly of the $B 61$.

13. Figure 6.1. Archimedes $3.0^{\oplus}$ generated cost analysis spreadsheet.

14. Figure 6.2. Archimedes $3.0^{\oplus}$ frame-grab to associate with Step 1080 in Figure 6.1.

15. Figure 7.1. The Small Smart Machine Design System.

\section{Tables}

1. Table 1.1. Constraints implemented in the Archimedes $3.0^{\odot}$ System.

2. Table 4.1. Additional constraints not provided in Chapter 1. constraints precedence over subassembly constraints. level constraints precedence over top-level constraints. subassembly constraints. and have no precedence. 
Intentionally Left Blank. 


\section{Executive Summary}

An assembly is a product consisting of two or more independently fabricated pieces, called parts; a subassembly is any nonempty subset of the parts of an assembly. An assembly plan for a product is a sequence of motions and manipulations of the parts that transforms the individual parts into the finished product. Given a complete description of an assembly, assembly planning is the problem of determining a feasible assembly plan for that product. Disassembly planning, in this context, is the planning of operations that take an assembled component apart. Feasibility and cost of disassembly operations differ greatly from their assembly counterparts for planning purposes. For instance, pressfits are rarely possible to disassemble without damaging one or both parts. Generative process planning describes the methods process engineers use to modify manufacturing (or process) plans after a design is complete. Closely related to assembly plans are disassembly plans, generative process plans and service plans, the latter being plans for partial disassembly and re-assembly.

In theory, a complete assembly plan includes every detail of the assembly process down to the factory floor, including the motions of all parts, humans, and robots that put it together and full descriptions of the fixtures, jigs, tools, etc. that are used. In practice, assembly plans are almost never written down in that much detail and at the very least, line workers decide their own body motions to assemble the product. The assembly process itself is its only true description. Instead, representations of assembly plans are abstractions: they specify the assembly plan down to a certain level of detail, and leave the corresponding complete plan to be determined. Automated assembly planners therefore must plan for assembly and disassembly at some level of abstraction. Because the planners do not reason about customer-specific manufacturing plan criteria, constraints, below their abstraction level, they often produce plans that, when those constraints are considered, are not feasible. The work conducted under the purview of this LDRD is intended as one step further down the abstraction hierarchy, to generate assembly and disassembly plans in more detail that are therefore more likely to be feasible. Alternatively, the resulting planner will require less human input in an interactive assembly planning system.

Constraints on assembly plans come from a wide variety of sources. Design requirements, part and tool accessibility, assembly line and workcell layout, requirements of special operations, and even supplier relationships can drive the choice of a feasible or preferred assemble or disassembly sequence. Designing products for easy assembly and disassembly during their entire life cycles for purposes including product assembly and disassembly, product upgrade, product and servicing and repair is a process that involves many disciplines. In addition, finding the best solution often involves considering the design as a whole and by considering its intended life cycle. Different goals and assembly plan selection criteria, constraints, as compared to initial assembly require re-visiting significant fundamental assumptions and methods that underlie 
current assembly planning techniques. Previous work in this area has been limited to either academic studies of issues in assembly planning or to applied studies of life cycle assembly processes that give no attention to automatic planning. It is believed that merging these two areas will result in a much greater ability to design for, optimize, and analyze life cycle assembly processes. Sandia's Automated Assembly Analysis System, Archimedes 3.0 $0^{\odot}$, is used as the foundation for integrating the automatic planning algorithms for later life cycle assembly processes.

In the Archimedes $3.0^{\oplus}$ System, users specify plan selection criteria in the form of constraints using a graphical user interface. This constraint-based interactive approach to automated assembly analysis and planning has proved extremely valuable in demonstrating manufacturability of a product when starting with the original CAD data. However, when addressing later life cycle assembly processes, the system has shown some deficiencies. To address the deficiencies of the system, the constraint framework has been extended to include planning for later life cycle assembly processes. For this reason, all of Chapter 1 is devoted to the assembly planning constraint framework implemented in Archimedes $3.0^{\circ}$.

Throughout the remaining chapters, the "constraints" concept will be continuously emphasized, either explicitly, implied by context, or by example. For example, Chapter 2 is devoted to Archimedes $3.0^{\circ}$, the foundation used to implement automatic planning algorithms for later life cycle assembly processes. Archimedes $3.0^{\odot}$ is a constraint-based, interactive assembly planning software system used to plan, optimize, simulate, and document sequences of assembly. Given a computer-aided (CAD) model (ACIS representation) of the product, the program automatically finds part-to-part contacts, generates collision-free insertion motions, and chooses assembly order. The engineer specifies a quality metric in terms of application-specific costs for standard assembly process steps, such as part insertion, fastening, and subassembly inversion. Combined with an engineer's knowledge of application-specific assembly process requirements, such as defining subassemblies, requiring that certain parts be placed consecutively with or before other parts, declaring preferred directions, etc., Archimedes $3.0^{\oplus}$ allows systematic exploration of the space of possible assembly sequences. The system considers thousands of combinations of ordering and operation choices in its search for the best assembly sequences and ranks the valid sequences by a quality metric. Graphical visualization enables the engineer to easily identify process requirements to add as sequence constraints. Planning is fast, enabling an iterative constrain-plan-view-constrain cycle.

All of the remaining chapters are devoted to the automatic planning for later life cycle assembly processes including product assembly, product disassembly, product upgrade, and product servicing. The revisions and extensions made to the planning algorithms and constraint framework of the Archimedes $3.0^{\circ}$ System for each area of study are presented.

Chapter 3 discusses automatic planning for disassembly in general. Planning the assembly and disassembly processes for a complex product is demanding and time-consuming, and costly mistakes are often made because the constraints cannot be reasoned about and resolved accurately enough or quickly enough. In planning for the assembly operations, a subassembly tree is based on insertion operations. An insertion operation is any single operation bringing together parts or subassemblies, or moving parts or subassemblies. It is a more general term than "insertion". In the Archimedes $3.0^{\circ}$ System, the insertion operations are called actions. Some of the methods 
describing how those actions are achieved include insert, threaded_insert, weld_insert, snapfit_insert, pressfit_insert, and glue_insert. Closely related to actions is the need to manipulate, attach, detach, and test parts and subassemblies using various hand and robotic tools. Constraints on assembly plans deriving from the need to use various tools in assembly or disassembly are called tool constraints. Often the optimal assembly plan is determined by tool constraints. In others, the main goal is to ensure assemblability and disassemblability with a given tool set that is already present in a facility or servicer's tool kit. Determining tool access constraints in complex geometric situations is particularly difficult for humans early in the design process unless costly (in time and money) prototypes are fabricated. Automated or partially automated methods to reason about tool constraints and assembly and disassembly processes help alleviate these problems. This chapter discusses the issues associated with reversing the insertion actions and presents the disassembly planning approach taken to reverse the insertion actions. Since many of the reversing operations require the use of tools, the Archimedes $3.0^{\circ}$ Tool Constraint Framework, which encodes tool accessibility constraints for various hand and robotic tools, was revised. The revisions to this framework, as well as the limitations of the framework are also discussed.

Chapter 4 focuses on the automatic planning for manufacturing generative processes. Generative process planning describes the methods process engineers use to modify manufacturing (or process) plans after a design is complete. A completed design may be the result from the introduction of a new product based on an old CAD design, an assembly or subassembly upgrade based on $\mathrm{CAD}$, or modified product $\mathrm{CAD}$ designs used for a family of similar products. This chapter describes methods that allow users to automatically combine assembly plans resulting from independent applications of Archimedes $3.0^{\Theta}$ to individual subassemblies of a larger assembly to form a complete assembly plan for the entire assembly. It further describes the implementation of constraint rules enabling a user to automatically "reconcile" existing constraints applied to an older version of an assembly to a new version of an assembly, which might have a different number (or ordering) of parts.

Chapter 5 discusses automatic planning for partial disassembly. The chapter covers the inclusion of a newly implemented constraint, REQ SUCCESS_PART(s), into the Archimedes $3.0^{\oplus}$ System to accommodate service-oriented assembly processes. The instantiation of a REQ SUCCESS_PART(s) constraint allows a user to specify a part or collection of parts that must be removed from an assembled product. This forces the planner to halt when none of the desired parts (as specified in the constraint) are left in the assembly. The chapter further describes a computer algorithm (and its implementation), which optimizes the search strategy for the REQ SUCCESS_PART(s) constraint. This search strategy uses a hill-climbing technique of the standard $\mathrm{A}^{*}$ search to repeatedly probe the subassembly tree, looking for better (less costly) disassembly sequences. An additional algorithm, referred to as the shortening algorithm, is also described. The purpose of the shortening algorithm is to "shorten" the initial sequence by eliminating unnecessary removal of parts not specified in the REQ_SUCCESS_PART(s) constraint set.

Chapter 6 focuses on the development of a computer tool to aid manufacturing engineers in their decision-making processes. It describes an integrated tool, the Design-for-Life-Cycle (DFLC) Cost Module, in which accurate cost estimates can be seamlessly derived from design requirements at the start of any engineering project. The chapter outlines the principles and 
implementation of the framework and how the user can interactively assist in the planning and optimization of assembly and disassembly sequences; and document assembly and disassembly processes and their associated costs. The result in using the DFLC Cost Module is faster cycle times through first-pass success; lower life cycle costs due to requirements-driven design and accurate cost estimates derived early in the process. In this work, emphasis was placed on automatic and computer-aided generation, optimization, simulation, and documentation, and "design-for" feedback of life cycle assembly plans derived using the Archimedes $3.0^{\circ}$ System.

Although somewhat disparate from the work described in the previous chapters on computeraided life cycle assembly analysis, Chapter 7 discusses the development and implementation of a computerized framework used to automate the design processes and automatically generate cost estimates for the manufacture of small vehicular robots, or small smart machines (SSMs). However, theoretically, constraints truly are the underlying principles. The system is written in $\mathrm{C}++$ and implemented in Tcl/Tk. The computer algorithms are designed to read in customerspecified mission requirements and generate a set of Sandia design requirements using a components database designed and developed using pre-existing SSMs at Sandia. The system then couples the datum to produce a cost-effective machine design based on a user-defined optimization criteria. Mission requirements include the following: type, payload, computational, operating environment, travel range, communication range, duty cycle, duration, dimensional footprint, quantity, budget, schedule, and optimization criteria. The Sandia design requirements include the following: chassis, motors, batteries, cameras, sensors, radios, pivot bearings, CPUs, and actuators. Optimization rules and calculations that are implemented include cost of the machine, weight of the machine, size in volume, and power consumption. 


\section{On the Automatic Generation of Plans for Life Cycle Assembly Processes}


Intentionally Left Blank. 


\section{Constraint-based Assembly Planning}

Constraints on assembly plans vary depending on product, assembly facility, assembly volume, and many other factors. This Chapter describes the principles and implementation of the Archimedes $3.0^{\Theta}$ Constraint Framework that supports a wide variety of user-specified constraints for interactive assembly planning. Constraints from many sources can be expressed on a sequencing level, specifying orders and conditions on part-mating operations in a number of ways. All constraints are implemented as filters that either accept or reject assembly operations proposed by the planner. For efficiency, some constraints are supplemented with special-purpose modifications to the planner's algorithms. Fast replanning enables a natural plan-view-constrainreplan cycle that aids in constraint discovery and documentation.

\subsection{Background and Motivation}

To be effective, computer-aided assembly planning systems must allow users to express their assembly and disassembly plan selection criteria that apply to their products and production environments. Because of its powerful geometric reasoning algorithms and assembly plan 
selection criteria framework, Archimedes@ 3.0 was selected as the foundation for which to build upon and implement disassembly planning algorithms for later life cycle manufacturing processes.

The Archimedes@ 3.0 System provides a library of assembly plan selection criteria, from which users can instantiate constraints on the assembly plan. Each constraint is described to the user in straightforward terms based on manufacturing and assembly sequencing concepts and defined using a simple graphical interface. However, Archimedes $3.0^{\odot}$ is limited to initial assembly and does not address, nor include constraints for, later life cycle assembly issues. This is a fundamental problem inherent in most assembly planners. Section 1.2 provides an overview of previous work addressing this and related problems. Section 1.3 details the concept of manufacturing plan selection criteria, now synonymous with "constraints". Section 1.4 closes the chapter with a moderately high-level overview of the Archimedes $3.0^{\odot}$ constraint framework.

\subsection{Previous Work}

Assembly planning systems that allow user-defined constraints have generally been of two types. In the first type $[5,6]$, users must specify all constraints before the long process of plan generation begins. In reality, this is rarely practical; the user finds it very difficult to list all constraints on assembly ordering until some possible plans are considered. In the second type of system (e.g. [2]), a large space of plans is first generated, and then undesirable operations and states are pruned interactively by the user. However, as assemblies become moderately complex the space of plans quickly becomes too large to edit or even generate. The methods implemented in the Archimedes $3.0^{\circ}$ System solve this problem interactively by generating one or a small number of assembly plans that satisfy the current set of constraints, and then allowing the user to impose additional constraints. Users can continue to add constraints and replan until a satisfactory plan is identified.

Previous efforts to incorporate a comprehensive set of user constraints in assembly planners were based on liaison precedence relations. Precedence relations specify logical combinations of part connections that must be established either before or after others. Precedence relations were pioneered by Bourjault [4] and greatly extended by DeFazio and Whitney [5]. Wolter et al [18] analyze the expressive power of precedence relations in detail. Precedence relations are quite powerful, but they can be very difficult to write correctly or understand as a user of an assembly planner. In Archimedes $3.0^{\odot}$ consideration was given to the idea of translating all constraints into precedence relations internally, but instead a procedural approach was adopted for reasons of efficiency and simplicity of implementation. The Archimedes $3.0^{\Theta}$ System demonstrates that an assembly planning system can achieve comprehensive constraint coverage while maintaining the advantages of a procedural representation. However, in planning for and optimizing life cycle assembly processes, there are substantial technical issues that preclude its use for maintenance, upgrade, and dismantlement applications, requiring fundamental rethinking of the approach to be used. 


\subsection{Manufacturing Plan Selection Criteria}

There are basically two categories of manufacturing plan selection criteria: assembly issues per se and planning issues. Assembly issues arise directly from physical constraints and manufacturing concerns, such as fixturing, manipulability, or issues of assembly line layout. Closely related are quality measures arising from a need to optimize one of the following basic aspects of assembly:
1) assembly cost;
2) time;
3) performance/reliability.

In contrast, planning issues are those that arise from the assembly process itself, such as simplifying assumptions and limitations of planning systems, or the need to handle large numbers of possible sequences practically.

The constraints on assembly plans vary depending on product, assembly facility, assembly volume, and many other factors. Assembly costs and other measures to optimize vary just as widely. Design requirements, part and tool accessibility, assembly line and workcell layout, requirements of special operations, and even supplier relationships can determine which orders of assembly are feasible. Among feasible orderings, a similarly diverse set of quality measures determines which is preferred or optimal. Together, the set of hard constraints and quality measures comprise a complex set of criteria that real assembly plans must satisfy. In Archimedes $3.0^{\circ}$, users specify manufacturing plan selection criteria in the form of constraints and quality measures. The constraints are implemented along the lines of the "assembly issues" classification. The Archimedes $3.0^{\circ}$ constraints framework is discussed in detail in the next section and provides background material for later life cycle manufacturing plan selection criteria presented in Chapters $2-6$.

\subsection{Assembly Planning Constraints}

The constraint framework described here was required for the Archimedes assembly planning system [10]. That system takes CAD data for a product as input and automatically determines geometrically feasible sequences of motions to assemble the product from its parts. However, geometry is only a small part of assembly planning. In [9], a survey of constraints that have appeared in the assembly planning literature was conducted, in hopes of adding many of those constraints to the Archimedes system. Two types of assembly constraints were identified. Strategic constraints apply to the entire assembly and its plan, while tactical constraints only apply to certain subsets of the parts. The result of the survey is an extensible library of simple but useful constraints that enable, highly interactive mode of assembly planning. Not all of those constraints, however, were chosen for implementation. The constraints that are implemented in the Archimedes 3.0 $0^{\odot}$ System were selected the following reasons:

User Friendly: Each constraint can be described simply in terms familiar to the user, has straight-forward effects, and combines with others in a very predictable way. 
Maintainable: Each constraint simply provides a filter procedure that disallows some assembly operations. The filter implementations are completely independent, allowing new constraint types to be added easily.

Efficient: Because the filters are procedures, they can be implemented in the most efficient way for that constraint. They test individual assembly operations, so they are compatible with standard state-space search and optimization methods. Furthermore, special-purpose methods can be added to improve efficiency.

The strategic constraints currently implemented in the Archimedes $3.0^{\oplus}$ System are flags for the planner. However, in theory there is no real difference between strategic and tactical constraints since tactical constraints can usually be applied to the entire assembly, and strategic constraints can always be limited to a subset of the parts. For instance, in [9], the REQ PATHS_AXIAL constraint is identified as strategic, is also very useful applied to a subset of the assembly. Implementing it as a tactical constraint allowed both uses, was simpler to implement, and caused no loss in efficiency. Table 1.1 lists the constraint types implemented in the Archimedes $3.0^{\odot}$ System.

Table 1.1. Constraints implemented in the Archimedes $3.0^{\oplus}$ System. Those marked by * have special-purpose routines for efficiency.

\begin{tabular}{|l|l|l|}
\hline Constraint Name & \multicolumn{1}{|c|}{ Purpose } & Scope \\
\hline REQ_CONNECT* & $\begin{array}{l}\text { Requires that every subassembly in the plan be connected. } \\
\text { This common constraint is implicit in cut-set methods such } \\
\text { as [1,8], }\end{array}$ & strategic \\
\hline REQ_LINEAR* & $\begin{array}{l}\text { Requires that parts be inserted one at a time [1,18]. This is } \\
\text { a common constraint as well as a common limitation in } \\
\text { other planning systems. }\end{array}$ & strategic \\
\hline REQ_VERTICAL* & $\begin{array}{l}\text { Requires that all parts be placed directly downward, but } \\
\text { unlike REQ PATHS_AXIAL, allows the assembly to be } \\
\text { reoriented so that any given action is considered } \\
\text { "downward." This constraint is very useful when planning } \\
\text { assembly with standard SCARA-type robots, where } \\
\text { insertions must be vertical but reorientations should be } \\
\text { minimized. }\end{array}$ & strategic \\
\hline Does not allow the assembly to enter a given state [2]. & tactical \\
\hline PRH_STATE & & \\
\hline
\end{tabular}




\begin{tabular}{|c|c|c|}
\hline Constraint Name & Purpose & Scope \\
\hline PRH_SUBASSY & $\begin{array}{l}\text { Prohibits use of a certain subassembly containing certain } \\
\text { part combinations. For example, one may need to avoid a } \\
\text { hard-to-fixture arrangement [6] containing a set of key } \\
\text { parts. }\end{array}$ & tactical \\
\hline REQ CLUSTER & $\begin{array}{l}\text { Requires that a set of parts be added to the assembly } \\
\text { consecutively, i.e., without interruption by other parts [3]. }\end{array}$ & tactical \\
\hline REQ FASTENER* & $\begin{array}{l}\text { Requires that certain parts be treated as fasteners for others } \\
\text { parts }[10,13] \text {. The fasteners must be placed immediately } \\
\text { after the fastened parts are mated. }\end{array}$ & tactical \\
\hline REQ LINEAR_CLUSTER & $\begin{array}{l}\text { A combination of REQ CLUSTER and } \\
\text { REQ LINEAR SUBSET. }\end{array}$ & tactical \\
\hline REQ LINEAR_PARTS & $\begin{array}{l}\text { Requires that a given set of parts be assembled linearly } \\
\text { when they are being mated with any of another set of parts. }\end{array}$ & tactical \\
\hline REQ_ORDER_FIRST & $\begin{array}{l}\text { Requires that the assembly plan start with a given part, such } \\
\text { as a "chassis" [11], or a set of parts. }\end{array}$ & tactical \\
\hline REQ ORDER_LAST* & $\begin{array}{l}\text { Requires that a specific part or set of parts be placed last in } \\
\text { the assembly plan. }\end{array}$ & tactical \\
\hline REQ_ORDER_LIAISON & $\begin{array}{l}\text { Requires some ordering between two or more liaison } \\
\text { creations; typically stated in a Boolean form such as } 1 \geq(2 \\
\text { and 3), or as a set of such Boolean statements involving } \\
\text { many liaisons [5]. This is a common and powerful type of } \\
\text { constraint, analyzed in [19]. }\end{array}$ & tactical \\
\hline REQ ORDER_PART & Requires an ordering between particular part insertions. & tactical \\
\hline REQ_PART_SPECIAL & $\begin{array}{l}\text { Any special-purpose part constraint, such as those dealing } \\
\text { with liquids, springs, snapfit parts, etc. }\end{array}$ & tactical \\
\hline REQ_PATHS_AXIAL & $\begin{array}{l}\text { Requires that each assembly action be along one of the six } \\
\text { coordinate directions of a given coordinate system, or a } \\
\text { selected subset of these six directions. Common special } \\
\text { cases are uniaxial constraints (allowing motions in either } \\
\text { direction along one axis) [12] and unidirectional constraints } \\
\text { [11]. This constraint is a limitation of many assembly } \\
\text { planning systems. }\end{array}$ & tactical \\
\hline REQ STACK & $\begin{array}{l}\text { Specifies a set of parts to be assembled one at a time in a } \\
\text { given direction. }\end{array}$ & tactical \\
\hline REQ_STAT & $\begin{array}{l}\text { Requires a set of parts to be in the stationary subassembly } \\
\text { when mated with any of another set. }\end{array}$ & tactical \\
\hline REQ SUBASSY* & $\begin{array}{l}\text { Requires that a particular subassembly be used in the plan } \\
{[1,6] \text {. }}\end{array}$ & tactical \\
\hline
\end{tabular}




\begin{tabular}{|l|l|l|}
\hline Constraint Name & \multicolumn{1}{|c|}{ Purpose } & Scope \\
\hline REQ SUBASSY_WHOLE* & $\begin{array}{l}\text { The same as REQ SUBASSY but tells the planner in } \\
\text { addition not generate a plan to construct the subassembly. }\end{array}$ & tactical \\
\hline REQ SUBSEQ & $\begin{array}{l}\text { Requires that a particular assembly subsequence be used } \\
\text { somewhere in the plan. This might be invoked because the } \\
\text { sequence is particularly efficient or reliable. The front-fill } \\
\text { then back-fill subsequences of [2] are relatively complex } \\
\text { examples. }\end{array}$ & tactical \\
\hline REQ TOOL* & $\begin{array}{l}\text { Requires that a collision-free placement of a given tool use- } \\
\text { volume must exist in the assembly during a certain } \\
\text { operation. See [17] for more details. }\end{array}$ & tactical \\
\hline
\end{tabular}

In the Archimedes $3.0^{\oplus}$ System, filters are an instance of generate-and-test, a standard paradigm in artificial intelligence [14] as well as in assembly planning [6, 8]. Attaching specialpurpose methods to constraint tests is also a well-known efficiency technique [14], although the methods used to accomplish this were novel.

A filter is nothing more than a simple procedure that accepts or rejects assembly operations. During planning, each proposed assembly operation is passed to the constraint's filter function, which returns true or false depending on whether the operation satisfies the constraint or not.

Only an operation that satisfies all current constraints is feasible. For instance, consider an operation placing subassembly $S_{1}$ into subassembly $S_{2}{ }^{1}$. The filter function of a REQ SUBASSY constraint with part set $P$ will return true if and only if

$$
\left.\left[P \subseteq S_{1}\right] \vee\left[P \subseteq S_{2}\right] \vee\left[\left(S_{1} \cup S_{2}\right) \subseteq P\right] \vee\left[S_{1} \cup S_{2}\right) \cap P=0\right]
$$

In other words, the operation satisfies the constraint if it keeps the parts in $P$ together, if only parts $P$ are involved, or if no parts in $P$ are involved.

As a standard interface to all constraints, the filter function provides a number of benefits. First and foremost, it makes the implementation of each constraint type independent. Interactions between constraints need not be considered, and each constraint can be implemented in its most straightforward and efficient way. This becomes crucial as the number of constraint types grows. In addition, constraints can vary in the data associated with them, their instantiation routines, various debugging outputs, and so on.

\footnotetext{
${ }^{1}$ An operation will typically have other specifications, such as a mating trajectory and perhaps an assembly orientation, but these are not relevant to REQ SUBASSY.
} 
For use in a standard state-space search method (such as generating an AND/OR graph for the assembly), it is important that the filter functions take as input single assembly operations, rather than more complex information such as a sequence of operations. In a state-space search, a given operation appears only once in the state graph and is either present or not. Hence, its feasibility cannot depend on operations that come before or after it. In the Archimedes $3.0^{\odot}$ framework, a number of constraints (e.g. REQ-SUBASSY above) must be implemented in a less natural way to apply to single operations than would otherwise be necessary.

Filter functions are flexible enough that a large subset of the constraints implemented in Archimedes $3.0^{\Theta}$, plus some additional ones that users requested are implemented. The flexibility is further demonstrated by the REQ TOOL constraint, [17], which encodes tool accessibility constraints for various hand and robotic tools within the framework.

Although filter functions themselves are usually quite fast, the generate-and-test abstraction can sometimes lead to an inefficient planning process overall. This is particularly true when many dead-ends appear in the search space or when a large number of assembly operations are generated, but few satisfy the constraints. In many cases, special purpose routines can increase efficiency dramatically. The constraint types for which special purpose routines have implemented are indicated with an asterisk $\left({ }^{*}\right)$ in Table 1.1.

\subsubsection{Interaction}

In experiments with product designers and assembly process engineers, it was found that a high level of interactivity is critical to the successful application of the assembly planner. Usually the designer cannot list all the constraints on assembly order at the start of the planning session. However, many of these constraints become "obvious" when the system graphically illustrates a plan that violates them. Seeing a violation, adding a constraint to remove that violation, and then replanning becomes the main cycle of interaction in the system. In this way, the assembly planner aids constraint discovery and management as well as plan generation and optimization.

Note, however, that placing a new constraint is very different from ruling out a certain operation, as performed in some previous systems such as [2]. Although a single operation demonstrates the need for a constraint, placing the constraint prohibits similar actions from occurring in many different operations, and hence limits the allowable plans far more than prohibiting a single operation. In the best case (and in many practical cases), the constraint encodes the manufacturing constraint exactly.

This plan-view-constrain replan cycle requires that replanning be performed at interactive speeds. In the Archimedes $3.0^{\oplus}$ System, a first assembly plan for a product can usually be found in a few minutes [10]. However, the most expensive part of planning is ensuring that part insertions are collision-free. By saving collision-detection information, replanning usually requires 10 to 20 seconds for assemblies of up to 100 parts.

There is, of course, no guarantee that all of the constraints the user has instantiated can be satisfied by a single plan. In this case, the planner fails and enters a "debug" mode that helps the user to determine the cause of the failure. If the constraints are all real, then a problem with the product design may be indicated. In most cases, some constraints can be adjusted to allow 
planning to succeed. When there are inaccuracies or inconsistencies in the product CAD data, planning can fail before the user has entered any constraints. The debug mode also supports finding such problems, and certain problems can be fixed within Archimedes 3.0

Note that if constraints are entered inaccurately, they may over-constrain the choice of plan and rule out some plans incorrectly. However, if some plans still satisfy the entered constraints, then the error may go unnoticed. This is a difficult problem to solve in a system allowing user specification of constraints.

After all known manufacturing constraints have been entered, the user can then ask for an optimal plan, according to user-specified costs of certain standard operations. In some cases, additional unstated constraints will be violated and discovered as the planner looks through a large space of plans to find the best. In this case, the new constraints must be added and the cycle repeats.

\section{References}

1. T. E. Abell, G. P. Amblard, D. F. Baldwin, T. L. De Fazio, M.-C. M. Lui, and D. E. Whitney. Computer aids for finding, representing, choosing amongst, and evaluating the assembly sequences of mechanical products. In [7], pages 383-435.

2. D. F. Baldwin, T. E. Abell, M.-C. M. Lui, T. L. De Fazio, and D. E. Whitney. An integrated computer aid for generating and evaluating assembly sequences for mechanical products. IEEE Trans. on Robotics and Automation, 7(1): 78-94, 1991.

3. N. Boneschanscher and C. J. M. Heemskerk. Grouping parts to reduce the complexity of assembly sequence planning. In E. A. Puente and L. Nemes, editors, Information Control Problems in Manufacturing Technology 1989: Selected Papers from the $6^{\text {th }}$ IFAC/IFIP/IFORS/IMACS Symposium, pages 233, 238. Pergamon Press, 1989

4. A. Bourjault Contribution à une approche méthodologique de l'assemblage automatisé: élaboration automatique des séquences opératoires. $\mathrm{PhD}$ thesis, Faculté des Sciences et des Techniques de l'Université de Franche-Comté, 1984.

5. T. L. De Fazio and D. E. Whitney. Simplified generation of all mechanical assembly sequences. IEEE Journal of Robotics and Automation, RA-3(6):640-658, 1987. Errata in RA-4(6):705-708.

6. J. M. Henrioud and A. Bourjault. LEGA: a computer-aided generator of assembly plans. In [7], pages 191, 215.

7. L. S. Homem de Mello and S. Lee, editors. Computer-Aided Mechanical Assembly Planning. Kluwer, 1991.

8. L. S. Homem de Mello and A. C. Sanderson. A correct and complete algorithm for the generation of mechanical assembly sequences. IEEE Trans on Robotics and Automation, 7(2):228-240, 1991.

9. R. E. Jones and R. H. Wilson. A survey of constraints in assembly planning. In Proc. IEEE Intl. Conf. On Robotics and Automation, pages 1525-32, 1996. 
10. S. G. Kaufman, R. H. Wilson, R. E. Jones, T. L. Calton, and A. L. Ames. The Archimedes 2 mechanical assembly planning system. In Proc. IEEE Intl. Conf. on Robotics and Automation, pages 3361-8, 2996.

11. $\mathrm{H}$. Ko and $\mathrm{K}$. Lee. Automatic assembling procedure generation from mating conditions. Computer Aided Design, 19(1):3-10, 1987.

12. E. Kroll, E. Lenz, and J. R. Wolberg. Rule-based generation of exploded-views and assembly sequences. Artificial Intelligence in Engineering, Design, and Manufacturing, 3(3): 143$155,1989$.

13. J. M. Miller and R. L. Hoffman. Automatic assembly planning with fasteners. In Proc. IEEE Intl. Conf. on Robotics and Automation, pages 69-74, 1989.

14. N. J. Nilsson. Principles of Artificial Intelligence. Springer-Verlag, 1980.

15. B. Romney. Atlas: An automatic assembly sequencing and fixturing system. In W. Strasser, R. Klein, and R. Rau, editors, Geometric Modeling: Theory and Practice, pages 397-415. Springer-Verlag, 1997.

16. R. H. Wilson. Minimizing user queries in interactive assembly planning. IEEE Trans. on Robotics and Automation, 11(2): 308-312, 1995.

17. R. H. Wilson. Geometric Reasoning about assembly tools. Technical Report SAND95-2423, Sandia National Labs, 1996.

18. J. D. Wolter, S. On the automatic generation of plans for mechanical assembly,"Ph.D. dissertation, Univ. Michigan, Ann Arbor, 1988. IEEE Trans.

19. J. D. Wolter, S. Chakrabarty, and J. Tsao. Mating constraint languages for assembly sequence planning. IEEE Trans. On Robotics and Automation. To appear.

20. J.D. Wolter and J. C. Trinkle. Automatic selection of fixture points for frictionless assemblies. In Proc. IEEE Intl. Conf. on Robotics and Automation, pages 528-534, 1994. 
Intentionally Left Blank. 


\section{The Archimedes $3.0^{\odot}$}

\section{Automated Assembly Analysis System}

Archimedes $3.0^{\oplus}$ is a constraint-based, interactive assembly planning software system used to plan, optimize, simulate, and document sequences of assembly. Given a computer-aided (CAD) model (ACIS representation) of the product, the program automatically finds part-to-part contacts, generates collision-free insertion motions, and chooses assembly order. The engineer specifies a quality metric in terms of application-specific costs for standard assembly process steps, such as part insertion, fastening, and subassembly inversion. Combined with an engineer's knowledge of application-specific assembly process requirements, such as defining subassemblies, requiring that certain parts be placed consecutively with or before other parts, declaring preferred directions, etc., Archimedes $3.0^{\odot}$ allows systematic exploration of the space of possible assembly sequences. The system considers thousands of combinations of ordering and operation choices in its search for the best assembly sequences and ranks the valid sequences by a quality metric. Graphical visualization enables the engineer to easily identify process requirements to add as sequence constraints. Planning is fast, enabling an iterative constrain-plan-view-constrain cycle. This constraint-based interactive approach to automated assembly analysis and planning has proved extremely valuable in demonstrating manufacturability of a product when starting with the 
original CAD data. However, when addressing later life cycle assembly processes, the system has shown some deficiencies. To address the deficiencies of the system, the constraint framework has been extended to include successful planning for later life cycle assembly processes.

This chapter provides a detailed view of the Archimedes $3.0^{\odot}$ System. The assembly planning approach is given in Section 2.1. Section 2.2 describes the graphical user interface. Section 2.3 covers situations when the planner might fail and describes how to recover. The efficiency of the system is provided in Section 2.4, while Section 2.5 concludes the chapter with an overview of the Archimedes $3.0^{\odot}$ implementation.

\subsection{Assembly Planning Approach}

The approach taken to assembly planning is obviously critical to the design, implementation, and performance of a user constraint system. It especially affects special-purpose routines for efficiency. The constraint system described in the previous section was added to the Archimedes mechanical assembly planner [2]. The system consists of four main elements: a user interface, a constraint system, a search engine, and an animation module. Archimedes $3.0^{\odot}$ generates twohanded monotone assembly sequences in reverse, starting from the more highly constrained, fully assembled state. This is a standard technique in assembly planning. The search space is an AND/OR graph of subassembly states and operations to construct them from smaller subassemblies. The planner uses a non-directional blocking graph (NDBG) of each subassembly [4] to efficiently determine assembly operations that might be performed to construct that subassembly, then checks these operations for geometric collisions, which is essentially a built-in filter. Operations are then checked against the list of user constraints.

The search strategy is carefully tuned to generate a first plan as quickly as possible in the domain of mechanical assembly. This is critical to achieve the desired plan-view-constrain-replan cycle of interaction. The search algorithm is not the focus of this report, and space allows only a cursory description. An AND/OR version of iterative sampling [3] is performed: during each pass of the algorithm, a single assembly sequence is generated, making random choices of operations to construct each subassembly. The first time any subassembly is visited, only a single operation is generated to construct it, and the known subassemblies of that operation are then visited. Bounds on quality measures for each subassembly and operation are stored and propagated in the AND/OR graph as they are generated. This allows useless search paths to be identified and pruned and an optimal plan to be identified when it is known. The strategy is designed to quickly reach a first solution, like a depth-first search, but to avoid getting caught by bad early decisions as a depth-first search would. The same algorithm functions as an any-time algorithm to optimize the assembly sequence when the user requests. 


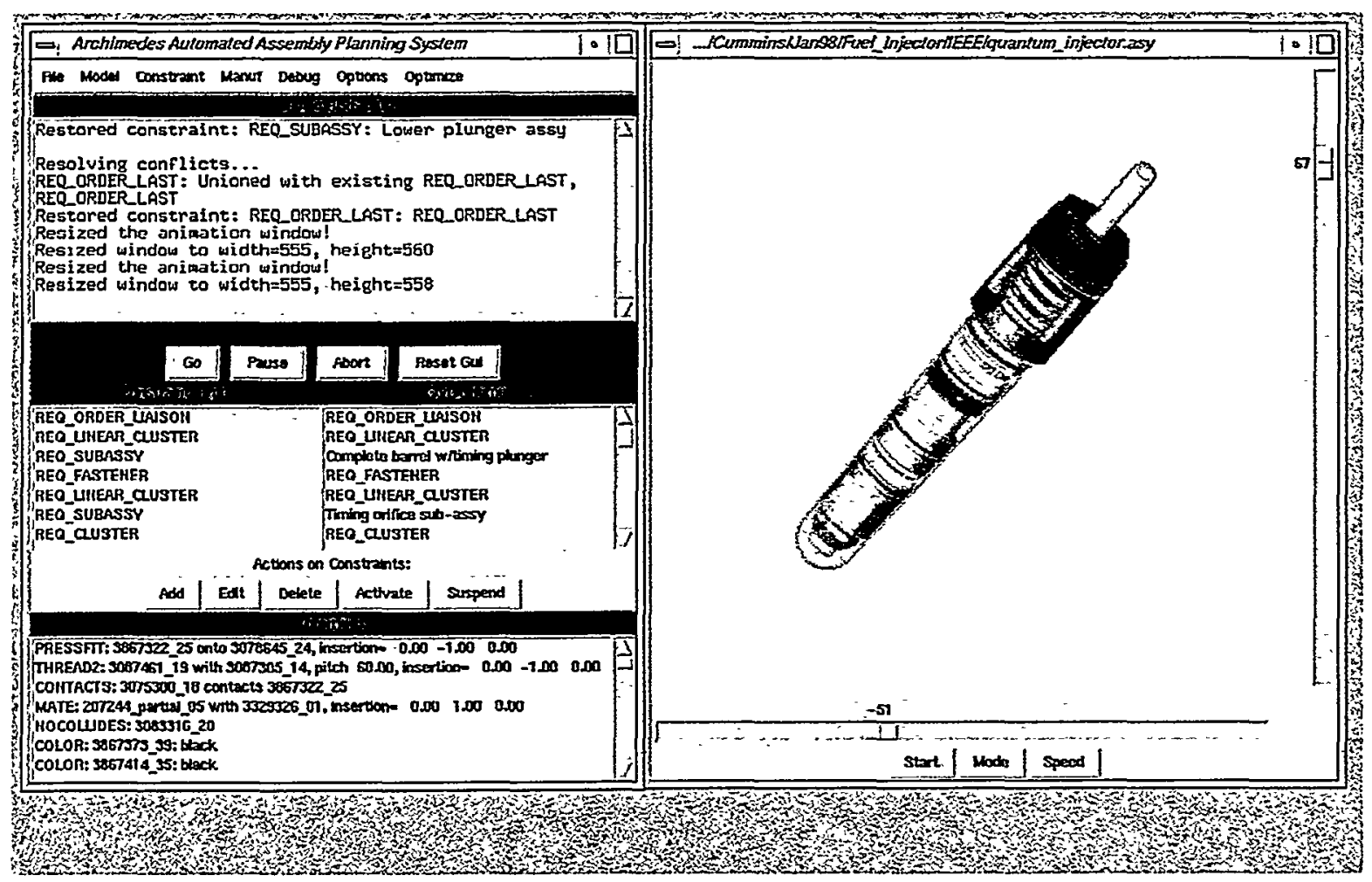

Figure 2.1: The Archimedes $3.0^{\odot}$ assembly planning system.

\subsection{User Interface}

The user interface is critical to effectiveness and user acceptance of an interactive planning system. The constraints must be easy to understand, define, and manage. In this section, features of the Archimedes $3.0^{\circ}$ user interface that are important to the success of the constraint system are described. See [1] for a better understanding of the Archimedes $3.0^{\odot}$ constraint interface.

Figure 2.1 (above) shows the main Archimedes $3.0^{\odot}$ user interface. The upper portion of the window on the left hand side serves and the main control window and displays the program's current status, displays any diagnostic output, and allows pausing or aborting any computation. The lower portion of the window on the left-hand side serves as the constraints and control panel and displays user-defined constraints and overrides. The window on the right hand side provides graphical output and part/subassembly selection. 


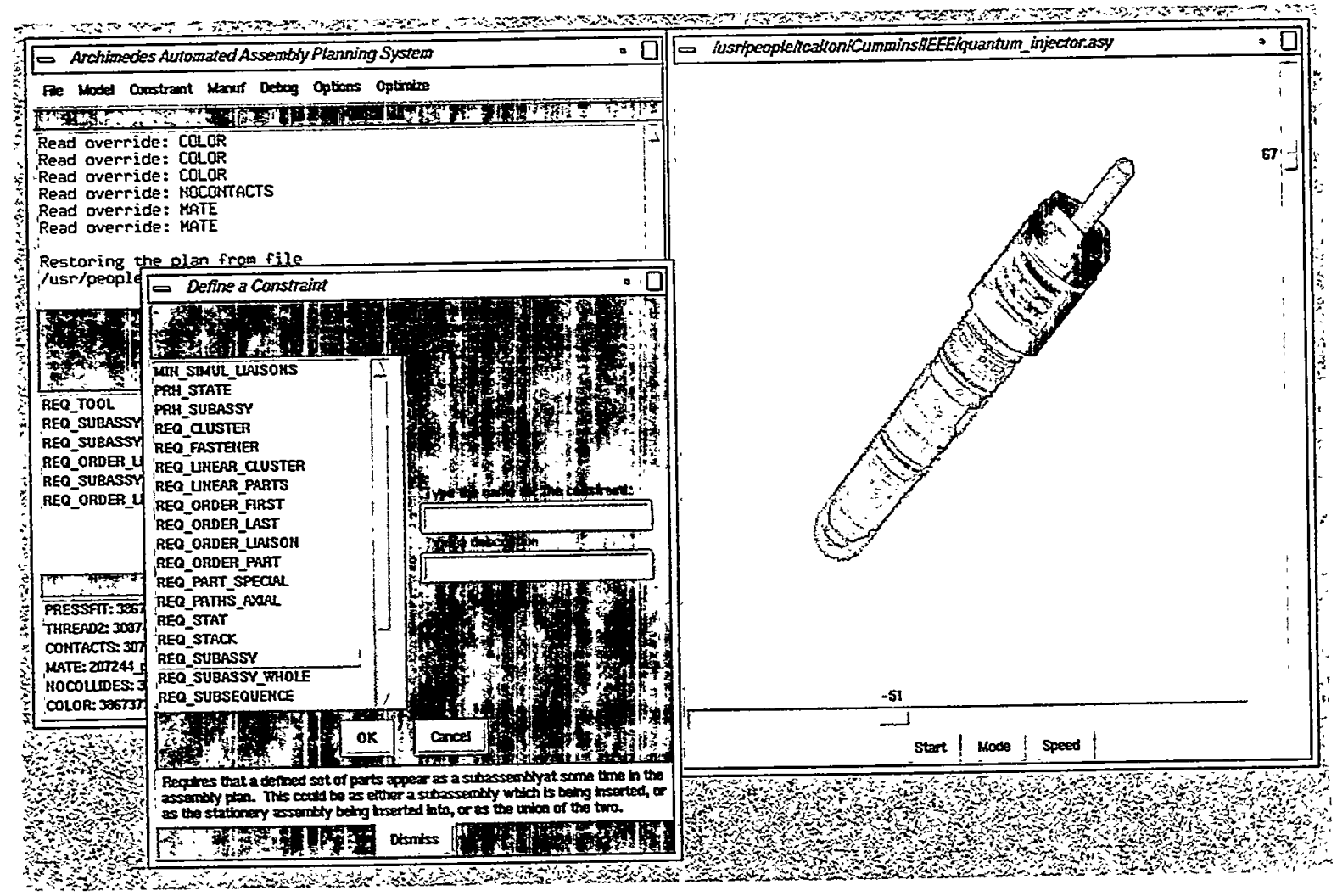

Figure 2.2: Planning dialog, showing a partial list of constraints.

Constraints are added by clicking on the "Add" button at the bottom, which brings up a sequence of menus and questions that let the user pick a constraint type and specify the particulars of the desired constraint. (See Figure 2.2.) Each constraint requires the user to select one or more parts of a "controlled" set in the graphical animation window, such as the parts making up a subassembly. An auxiliary window provides a list of named subassemblies to facilitate selection of larger sets of parts. (See Figure 2.3.) The user can give each constraint a name and descriptive comment. Some simple checks are performed to catch certain obvious inconsistencies within and between constraints.

Figure 2.3 shows the instantiation of a REQ SUB_WHOLE. In this setting, the user selects the parts using a point-and-click method from within the animation window shown on the righthand-side. The user is offered several access methods to selecting parts, which may be "inside" of the assembly. These options are shown at the bottom of the animation window on the next page. 


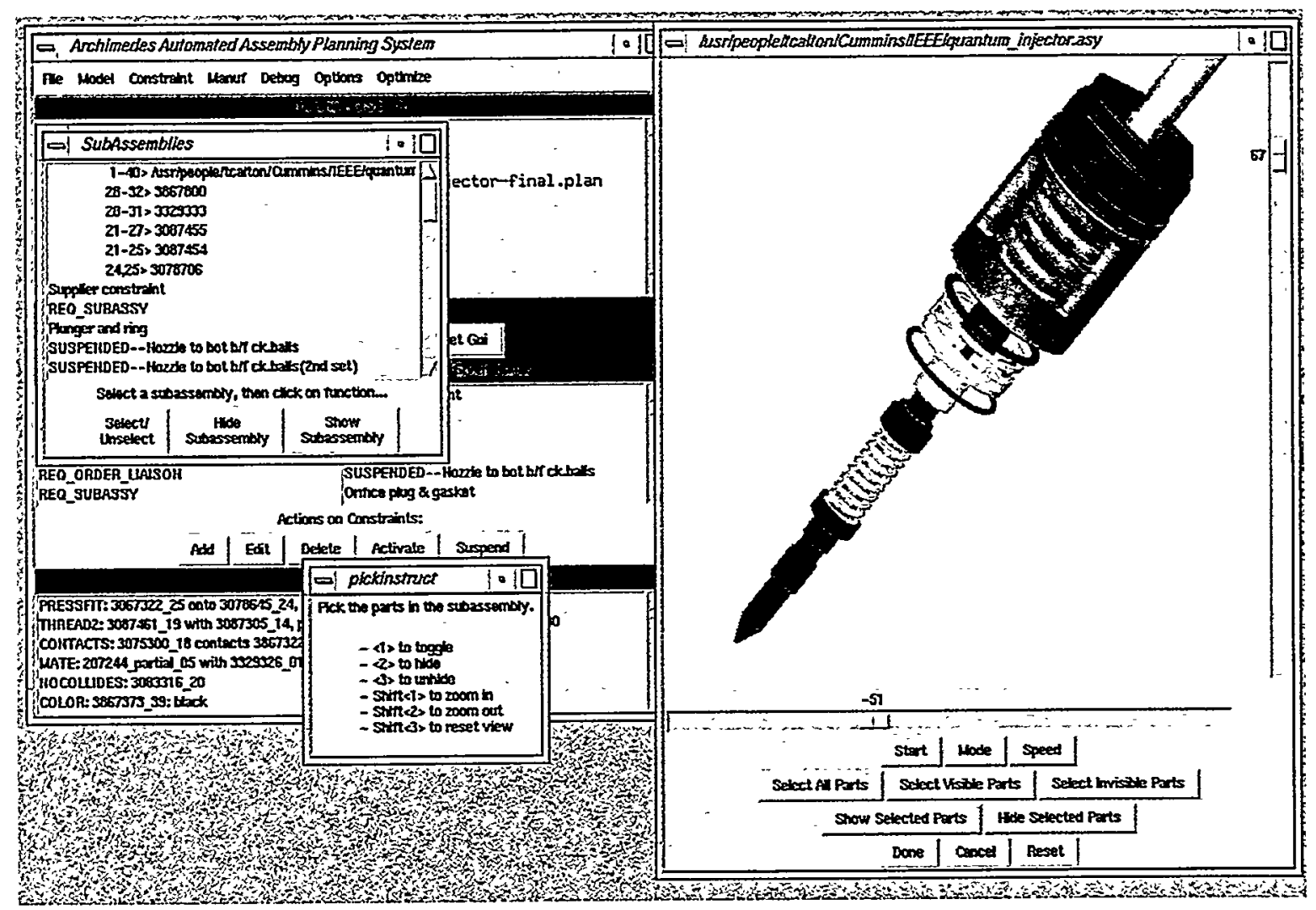

Figure 2.3: Planning dialog, showing the instantiation of a REQ SUB_WHOLE constraint.

Once defined, constraints are listed in the planning dialog (as shown in Figure 2.1). They can be edited using a process very similar to initial definition. They can also be deleted, temporarily suspended, and re-activated. Constraint suspension is a very useful feature that allows the user to consider various scenarios for assembly. Constraints often embody assumptions about the product assembly scheme; by suspending some and replanning, the user can compare the cost of removing the assumption to the possible gains in assembly sequence efficiency that result.

\subsection{When Planning Fails}

When a product cannot be assembled according to the current set of constraints, the planner fails and enters a "debug" mode that helps the user to determine the cause of the failure. For example, one can request that the planner try to remove a particular part or subassembly (from the subset of parts remaining when the planner failed) in a direction that appears possible. Collisions or constraints that disallow the operation are then posted in the status window. Other options 
include trying to disassemble every pair of parts in the offending subassembly, or trying to remove any parts along a given trajectory. This constraint debugging capability is very important and useful, since in some cases it may not be apparent to the user why the planner cannot find a feasible plan.

Often, the planner can fail without any user-defined constraints. This is sometimes due to limitations in the planner's algorithms, such as an inability to reason about flexible parts such as snapfits and springs. Other times, inaccuracies or inconsistencies in the product CAD data cause the planner to fail. Examples include pressfit parts and threaded parts that are modeled as cylinders too large for their holes. Archimedes $3.0^{\odot}$ includes a set of model adjustment features or overrides, which can be used to correct such problems. These include a function to effectively add threads to cylindrical contacts between parts; to specify that certain part insertions are in fact possible, even though collisions occur between the parts; and to delete a part temporarily.

\subsection{Efficiency}

As mentioned above, the generate-and-test abstraction can sometimes lead to an inefficient planning process where a large number of operations are generated, very few of which pass through the filters. In such cases, supplemental routines can improve planning efficiency greatly. These routines are very dependent on the internal algorithms of the planner. Because Archimedes $3.0^{\circ}$ plans backward from the assembled state to individual parts, the supplemental routines must be implemented in reverse.

For instance, if the user has created a REQ SUBASSY constraint with part set $P$, and parts not in $P$ are present, then $P$ cannot be "split" at that point in the plan. To implement this constraint efficiently, a supplemental routine binds the parts of $P$ together for that stage, not considering any operations that split them. This is accomplished by placing bi-directional arcs between every pair of parts in $P$ in every blocking graph of the subassembly [4].

Supplemental routines must be considered carefully, trading off the added speed against the increase in planner complexity. Three characteristics identify candidates for supplemental routines:

1. The constraint either leads to many dead-ends in the search space or rules out a very large proportion of generated operations,

2. An efficient method exists to implement the preprocessing, and

3. The constraint is used often.

The REQ FASTENER constraint type is another instructive example. Fasteners are very common in mechanical assemblies. The REQ FASTENER constraint requires that as soon as one set of parts is joined (the fastened parts), and then a set of fastener parts must immediately be placed. In reverse, this constraint means that as soon as a single fastener part is removed, then all other fasteners must be immediately removed, followed by at least one of the fastened parts. If 
any of the fasteners cannot be removed, a dead end appears in the search space (in fact many can appear).

The filter function for REQ FASTENER is straightforward, but its supplemental routines are the most complex implemented. The fastener parts are removed from the assembly representation and considered secondary parts that implicitly must be added when the fastened parts are mated. Before generating operations to construct a certain subassembly, the planner determines which fasteners could be placed into the subassembly. For each fastener that cannot be placed, the corresponding fastened parts are bound together as for REQ SUBASSY. Fasteners are placed back in subassemblies for collision checking and other calculations.

Note that when a constraint has supplemental routines, the planner still calls the constraint's filter function, which should never return false. This double check is very useful to ensure correctness, because the supplemental routines are complex and interact with each other. It is conceivable that a supplemental routine would only reduce the number of operations rejected by the filter function, but we have not found such a case.

In almost all cases, adding constraints reduces planning time. The reduced size of the search space usually outweighs the extra time required to compute the filter functions. In fact, constraints can be used to guide the planner to a correct plan for assemblies that would otherwise have intractably large search spaces.

\subsection{Implementation}

Archimedes $3.0^{\odot}$ is implemented in $\mathrm{C}+$, with $\mathrm{Tcl} / \mathrm{Tk}$ used for the graphic interface and OpenGL ${ }^{\mathrm{TM}}$ for 3D graphics and animation. The constraints are implemented as a hierarchy of $\mathrm{C}++$ derived classes. Each type of constraint simply overrides the filter function from a base class, along with methods to define the type, name, etc. of the constraint. Each constraint type also has its own data members, such as part sets, tool choices and points of application, and so on. Some of the supplemental routines are implemented as constraint class methods; however, most cannot be separated from the planner's algorithms, and are woven directly into the planner implementation.

\section{References}

1. R. E. Jones and R. H. Wilson. An interactive assembly planning system. In Video Proc. IEEE Intl. Conf. on Robotics and Automation, 1997.

2. S. G. Kaufman, R. H. Wilson, R. E. Jones, T. L. Calton, and A. L. Ames. The Archimedes 2 mechanical assembly planning system. In Proc. IEEE Intl. Conf. on Robotics and Automation, pages 3361-8, 2996. 
3. P. Langley. Systematic and nonsystematic search strategies. In Artificial Intelligence Planning Systems: Proc. of the First Intl. Conf., 1992.

4. R. H. Wilson and J.-C. Latombe. Geometric reasoning about mechanical assembly. Artificial Intelligence, 71(2):371-396, 1994. 


\section{Automatic Planning for Complete Disassembly}

Many manufacturing companies today expend more effort on upgrade and disposal projects than on clean-slate design, and this trend is expected to become more prevalent in coming years. However, commercial CAD tools are better suited to initial product design than to the product's full life cycle. Computer-aided assembly analysis and planning, optimization, and visualization of life cycle assembly processes based on the product CAD data can help ensure accuracy and reduce effort expended in planning these processes for existing products, as well as provide design-for-life-cycle analysis for new designs. However, to be truly effective, the computer-aided assembly analysis and planning systems must allow users to express their assembly and disassembly plan selection criteria that apply to their products and production environments during the planning sessions.

Assembly tools are implements used to manipulate, attach, and test parts and subassemblies during the processes of assembly and disassembly. Tools in this sense include manual tools such as screwdrivers and hammers, robotic tooling such as welders and bolt drivers, and a number of other such items such as laser welders, riveters, and part-manipulation devices used in more traditional automation. If standard tools cannot be used to implement a given assembly plan, then 
a different plan must be chosen, the product must be redesigned, or special-purpose tools must be fabricated. Such special-purpose tools can be quite expensive, especially when they must be distributed to and stored at every in-field service center for the product. Taking tool constraints into account early in product design minimizes the need for such tools.

In many cases process engineers find it difficult to determine tool accessibility for assembly operations. This is especially true in cramped spaces containing geometrically complex parts that may interfere with the operation, such as inside the engine compartment of automobiles, ships, or aircraft. The problem is exacerbated by products designed by a large group of designers. Even when humans can accurately determine the feasible uses of tools, automated assembly planners that include tool constraints produce assembly plans with less human effort, allowing the results to be used earlier and more often in production realization and design-for-assembly.

\subsection{Introduction}

Planning the assembly and disassembly processes for a complex product is demanding and time-consuming, and costly mistakes are often made because the constraints cannot be reasoned about and resolved accurately enough or quickly enough. In planning for the assembly operations, a subassembly tree is based on insertion operations. An insertion operation is any single operation bringing together parts or subassemblies, or moving parts or subassemblies. It is a more general term than "insertion". In the Archimedes $3.0^{\odot}$ System, the insertion operations are called actions. Some of the methods describing how those actions are achieved include insert, threaded_insert, weld_insert, snapfit_insert, pressfit_insert, and glue_insert. Closely related to actions is the need to manipulate, attach, detach, and test parts and subassemblies using various hand and robotic tools. Constraints on assembly plans deriving from the need to use various tools in assembly or disassembly are called tool constraints. Often the optimal assembly plan is determined by tool constraints. In others, the main goal is to ensure assemblability and disassemblability with a given tool set that is already present in a facility or servicer's tool kit. Determining tool access constraints in complex geometric situations is particularly difficult for humans early in the design process unless costly (in time and money) prototypes are fabricated. Automated or partially automated methods to reason about tool constraints and assembly and disassembly plans help alleviate these problems.

This chapter discusses the issues associated with reversing the insertion actions and presents the disassembly planning approach taken to reverse them. Since many of the reversing operations require the use of tools, the REQ TOOL constraint framework, which encodes tool accessibility constraints for various hand and robotic tools was revised. The revisions to this framework are also discussed.

In the next section, the automatic generation of plans for life cycle assembly processes is placed in the context of previous research on constraints and assembly planning. Section 3 gives an overview of the tools constraint framework implemented in Archimedes 3.0 $0^{\circ}$. Finally, Section 4 associates the tools constraint framework with insertion actions (and their reversal) and presents the disassembly planning approach taken to reverse the insertion actions. 


\subsection{Previous Work}

\subsubsection{Disassembly Planning Constraints}

Chapter 1 presents an overview of the Archimedes $3.0^{\oplus}$ constraint framework. In Section 1.2, the framework is placed in the context of previous efforts in planning for assembly with constraints. However, in planning for and optimizing life cycle assembly processes, there are substantial technical issues that preclude its use for maintenance, upgrade, and dismantlement applications, requiring fundamental rethinking of the approach to be used. Some of those issues include disassembly operations, partial assembly, non-monotonic assembly, destructive disassembly, and dismantlement and disposal. A perhaps less technical issue than these, yet just as important, is how to most effectively feed the system's results back to the designers to enable design-for-life-cycle.

\section{Disassembly operations}

The mechanics of disassembly operations must be characterized as to feasibility and cost, and differ greatly from their assembly counterparts for planning purposes. For instance, pressfits are rarely possible to disassemble without damaging one or both parts, which is sometime acceptable.

\section{Partial assembly}

Assembly and disassembly do not always proceed from or result in individual parts. For instance, a field upgrade may only require only partially disassembly of a component, and then subassemblies are placed in it. Related research outside assembly planning is more extensive. Programs from Boothroyd-Dewhurst do enable design-for-service and recycling by analyzing plans entered by the user, but do no planning or optimization. Finally, researchers in concurrent engineering and green engineering have studied designfor-service and design-for-disposal (for instance $[2,5,20]$ ), but lack of assembly planning capability limits them to heuristic and statistical methods.

\section{Non-monotonic assembly}

In the assembly planning literature, operations are non-monotonic when they leave parts in intermediate positions rather than placing or removing them completely. For instance, removing three screws from an access plate and leaving it hanging on the fourth screw is non-monotonic. Non-monotonic assembly planning is the most difficult issue computationally and is known to be PSPACE-hard [25], and the only system to generate such plans was limited to an impracticably small number of parts [11].

\section{Destructive disassembly}

In some applications, operations that destroy parts (i.e., cutting, tearing, or melting) are acceptable in disassembly. Destructive operations have complex and sometimes unpredictable geometric effects. However, in many cases they are the most effective method of disassembly. The only known study of planning methods for destructive 
disassembly used a simplified model of destructive operations that does not correspond to what is seen in practice [8].

\section{Dismantlement and disposal optimization}

This problem has an erratic cost criterion, since a small amount of disassembly sometimes makes huge gains in recovery/disposal gains/costs. This makes optimization computationally difficult compared to initial assembly (which has a non-decreasing cost function). The commercially available RESTAR System [20] attempts to optimize disassembly processes for recycling and is based on a service-assembly planner described in [21]. Both rely on user input to determine all possible operations, making them impractical on products of more than ten to twenty parts.

\subsubsection{Tool Constraints}

Previous work related to representing and reasoning about tool constraints can be roughly divided into five parts: assembly sequencing work on "attachments," planning for machine tools, general-purpose reasoning about tools, special-purpose planner for specific tools, and work on estimating the difficulty of fastening operations when obstructions are present.

\section{Assembly Sequencing and Attachments}

The past decade has seen a great deal of research on assembly planning and related topics (see, e.g., $[1,6,13])$. Most focuses on graph-theoretic techniques and constraint languages and on collision avoidance. However, much of this research points to a need for more rigorous and powerful method to reason about tool constraints.

For instance, Homem de Mello and Sanderson $[12,14]$ use a relational model of assemblies as the basis of their assembly sequencing approach. The relational model includes attachments between parts in the assembly, which correspond to fastening operations. Each attachment contains a list of parts whose presence prevents the attachment from being accomplished. However, $[12,14]$ do not address how to determine which part prevent which attachments.

Henrioud and Bourjault [10] use a similar product model, including attachment information. However, their model does not represent parts that prevent attachments; instead, they defer to the expert judgement of an engineer to determine the feasibility of an attachment in a given subassembly. The answer is stored in a database of constraints on the assembly. This can cause many hundreds of questions to be asked of the engineer, some of which can be very difficult to answer accurately in complex assemblies. As a result the system cannot reasonably be applied to assemblies with more than about 20 parts.

Miller and Hoffman [18] describe a system that requires access space above screws, bolts, and nuts before they can be removed. However, the tests used to determine access are very simple, consisting of a ray casting and box tests, and only approximately distinguish between feasible and infeasible tool applications. It is also unclear how these tests could be generalized to other tool requirements. 


\section{Machine Tools}

Reasoning about the effects and use of machine tools is a well-studied problem ([22] is a good but slightly out-of-date survey). GARI [ 6 ] was an early example of a machining expert system, compromising among pieces of advice to order machining operations, including choosing tools to accomplish them. A recent comprehensive system is described in [19]. Practical methods to help generate tool paths and check machining plans have matured to the point that they are now regularly used in commercial CAD/CAM packages such as AutoCAD and Pro/ENGINEER.

However, the constraints applying to the use of machine tools have little in common with assembly tools. Machine tools are essentially subtractive in their effect - always removing material - and are much more homogeneous than assembly tools, which vary widely in shape, purpose, and use. Furthermore, the constraints on machining include such issues as cutting forces, fixturing, satisfying tolerances, and material deformations that rarely appear in the same form in assembly. Finally, machine tools are, in the main, applied to single parts rather than assemblies, and do not affect the assembly.

Note that in some cases a machine tool is applied to multiple assembled parts, such as when parts are aligned and then holes are drilled for fasteners. These operations are common in industries such as aircraft construction and shipbuilding. We consider any tool applied to assembled parts to be an assembly tool, and hope to handle these cases in our approach.

\section{General-purpose Tool Reasoning and Recognition}

Initial work on an ambitious system to reason about tools and their uses was reported by Brady et al. [4]. Their system, called "Mechanic's Mate" was to recognize tools from images, determine their uses analogically or from first principles, and even design new tools automatically. However, the project was canceled before significant progress could be made. See [9] for a more recent effort along these lines.

\section{Special-purpose Planners}

Special-purpose systems have been created to reason about specific tools and plan their use. For instance, in [23], the position and approach path are planned for a coordinate-measuring machine. Determining visibility regions for a camera (in our definition, a camera is a tool when used to facilitate or inspect and assembly operation) is closely related to aspect graphs in computer vision [17], to which our methods have some mathematical similarities. Miller and Hoffman's constraints on accessibility of screws and bolts fall into this category as well [18].

\section{Quantifying Obstructed Access}

Experiments have been performed on the time workers take to execute certain mechanical fastening operations under varying conditions [3]. The operations studied including screwing, nut tightening, and pop riveting, using a variety of tools and under conditions ranging from normal to obstructed access and restricted visibility conditions. Díaz-Calderón et al. [7] present progress toward automatically determining the difficulty of using a screwdriver in a particular assembly operation. They assign costs to the 
available access angle, operation angle, and clearance for the hand around the screwdriver, and compare these to the values given in [3].

\subsection{Geometric Reasoning about Assembly Tools}

Planning for assembly, servicing, and disassembly of a product is a critical step in the design realization process for that product. In addition, assembly planning can supply important feedback to help designers improve the design from a manufacturing standpoint. Since the need to manipulate, attach, detach, and test parts and subassemblies leads to important constraints on assembly and disassembly plans, methods must be developed to reason about these constraints. This Section summarizes the approach to cover a wide variety of such constraints and describes an implementation of the approach in the Archimedes $3.0^{\Theta}$ System.

\subsubsection{Representing Tools}

The representation of tool constraints is divided into (1) information about a tool independent of any assembly, and (2) information about an application of a tool in a particular assembly. The information specific to a tool consists of the following three parts. A tool's relative time specifies whether the tool is applied before, during, or after the actual mating of parts in an operation. A use volume is a minimum region of space that must be free in an assembly to effectively apply the tool. Finally, the placement constraints are constraints on where the use volume can be located relative to the parts the tool must act on. Together, these three pieces of information define a canonical tool. This section describes the representation of canonical tools in more detail. The next section describes tool applications, which specify a particular need for a tool in an assembly.

Geometric tool constraints have been implemented in the Archimedes $3.0^{\odot}$ assembly analysis and planning system. Only 0-degrees of freedom (DOF) and 1-DOF feasibility predicates have been implemented to date; these apply to pre-tools, in-tools, and post-tools. The 1-DOF predicates are limited to a single rotational degree of freedom, which is the most common type of placement freedom found in mechanical assembly tools. Because of the complexity of implementing the exact combinatorial algorithms, 1-DOF predicates instead test a fixed number (16 at present) of sample values of the free angle. For each application, the user can choose either of two routines to test for intersection of a use-volume with other parts in the subassembly. The first uses ACIS solid geometry intersection routines. The second is discretized test employing the Zbuffer of a 3D graphic-accelerated workstation (see [15] for more details). The latter is much faster, but requires that there be a vertical path for insertion of the use-volume into the assembly (i.e., along the z-axis of the application transform). Lazy evaluation is used for both feasibility and placing arcs in the blocking graphs for infeasible post-tools.

A library of 124 manual, robotic, and miscellaneous assembly tools, chosen for their relevance to Sandia applications has been constructed. The tool library covers 11 mechanical tools types (e.g., wrenches, screwdrivers, ratchets, etc.), a laser welder and two types of robotic grippers.

The tool library is organized as an object hierarchy to take advantage of similarities between tools in both representations and reasoning techniques. For example, the non-offset screwdrivers, 
socket drivers, and full-turning hex keys are all instances of the same class. Their relative times and placement constraints are the same; the only differences are the tool and use-volumes.

Similarly, offset screwdrivers, wrenches, and partial-turning hex keys are closely related. The top level of the hierarchy breaks down by degrees of freedom, followed by relative time. Each tool also has routines to create tool-level animations of assembly plans. Because the volumes for each tool require large amounts of memory, they are only loaded when a constraint using the tool is defined.

Tool constraints are defined using the graphical interface outlined in Chapter 2. The user selects the target part set using the mouse and chooses the tool from a hierarchical listing of canonical tools. A primary part is then chosen if the tool requires one. Finally, the user defines the application transform by graphically positioning the tool's reference frame with respect to the target parts. If the tool is a 1-DOF, the user has the option of specifying the value of the degree of freedom, rather than letting it vary. This effectively makes the $0-\mathrm{DOF}$ for that application. Figure 3.1 shows the application transform being defined for a socket wrench. Finally, alternate tool applications can be specified to accomplish the same assembly purpose. The alternate applications have the same target part set, but all other details are independent. An operation is feasible if at least one of the alternate applications is feasible.

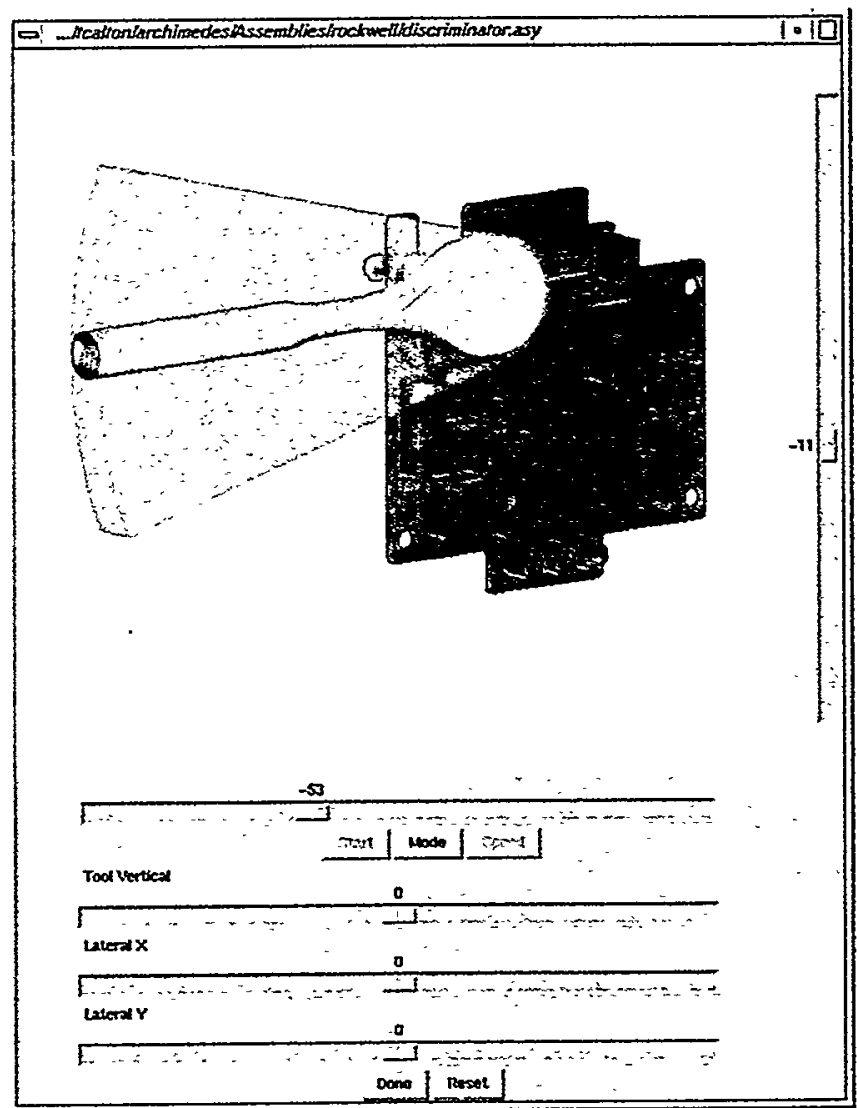

Figure 3.1. Defining the placement of a socket wrench for a REQ TOOL constraint. The constraint requires the translucent volume to be free when the tool is applied. 


\subsection{Disassembly Planning Approach}

\subsubsection{Disassembly Operations}

In the case of destructive and non-destructive disassembly, new actions were implemented into Archimedes $3.0^{\circ}$ System. An action is defined to be any single operation of bringing together parts or subassemblies. It is a more general term than "insertion". These actions were propagated to the various load, save, animate, etc. routines that need to use them. The current full list of actions is as follows.

For assembly, the actions include

INSERT, THREADED_INSERT, WELD_INSERT, SNAPFIT_INSERT, PRESSFIT_INSERT, and GLUE_INSERT

For disassembly, the actions include:

REMOVE, THREADED_REMOVE, UNWELD, UNSNAPFIT, UNPRESSFIT, and, UNGLUE

For destructive disassembly, the new actions include:

CUT, SHRED, REUSE, LANDFILL, TOXIC_DISPOSAL, and RAD_DISPOSE

A further note on this subject is that "UNSNAPFIT" and "UNGLUE", and to a lesser extent, "UNPRESSFIT" and "UNWELD" are, operationally, somewhat ill defined concepts at this time. This is necessitated by the fact that the reversing of SNAPFIT AND GLUE operations cannot be readily defined in the absence of information about the parts involved. For example, some snapfit operations can be undone readily, with the application of a custom tool which, spreads the tabs on the snapped-into part so that the snapping part can be removed without damage to either part. A similar operation, thought, might be irreversible without destructive effect on the parts -- if the tabs previously referred to are inaccessible once the snapping part has been put in place, for example. Similarly, with some types of glues and adequate accessibility, a glue operation might be reversed by application of a hot-knife, while with other glues or part geometry's, nondestructive ungluing might be impossible. Cost criteria has been employed that assume destructive disassembly, and allow the user to specifically lower the costs in cases where nondestructive disassembly were both desired and possible.

\subsubsection{Disassembly Planning with Tools}

The tools framework was also revised. The intent was that the tools, rather than being applied in an arbitrary fashion, be applied in a manner consistent with the operations they support. Thus, 
by specifying that an operation is a "Weld Operation", one specifies/restricts that if a construction tool is applied, it must be a welder, and that if a destruction tool is applied, it must be a cutting torch, or similar tool. The specific organization of the revised framework is as follows:

\section{Overrides}

Overrides were reduced to completely non-manufacturing related issues. That is, contact and collision analysis, faceting parameters, color, and mate overrides, which were once handled as manufacturing issues, are now handled separately. These types of overrides relate specifically to inconsistencies in data due in part to translation issues (as opposed to, e.g., mates requiring user intervention because of intentionally tight/nonexistent tolerances for pressfits).

\section{Constraints}

Constraints remained essentially unchanged, except that the REQ TOOL constraint is now either rendered an anachronism, or modified to check action types before allowing use of a particular type of tool. The "Manuf" constraint options pick up the threaded mate/unmate actions, as well as dismantlement operations and any other assembly/disassembly actions not currently listed, which might be added in the future. Specification of a manufacturing operation now includes the optional specification of tool applications.

A corollary effect of this framework was to make the framework easier to apply assembly and disassembly costs. The Archimedes $3.0^{\odot}$ System would apply generic assembly and disassembly costs based on the action type, if no other information were available. If the tool selection was available, this would refine the cost values somewhat. For a better estimate, the user is still allowed to provide specific costs for a given operation on two parts.

\subsubsection{Limitations of the Approach}

The primary question regarding the use of tools is "Is there space for this tool to be used?" This is the main question product designers have struggled with for years. The framework implemented in Archimedes $3.0^{\oplus}$ attempts to capture as much of the issue as possible in a practical way. Although the framework does not cover many other questions, one might wish to ask about geometric reasoning for tools in the automatic generation of plans for life cycle assembly processes. However, the framework does achieve broad coverage and lays the foundation for further extension to address those other questions.

Among the issues that are not addressed in the tools framework are:

\section{Determining Applications}

Many tool applications are specified in the assembly design (such as weld points and press fits), or can be inferred easily from the CAD data (for instance, that a screwdriver is needed to thighten each screw). Beyond that, automatically determining required tool applications become a difficult feature-recognition task.

\section{The Tool Wielder}

Reasoning about the spatial requirements of the agent wielding the tool (a robot or human arm, or hard automation) is beyond the scope of this work. Human hands in particular are extremely flexible, and determining automatically whether they (or mechanical tool wielders) will 
be able to reach and manipulate tool is very complex (see e.g. [16]). As a result, the tool constraints considered in this framework are necessary but not sufficient constraints on tool-level assembly plans.

\section{Mechanics}

Which tools can accomplish a task often depends on non-geometric issues such as the force that must be applied, accuracy required, expected deformation forces, possible damage to the parts, and so on.

\section{Optimal Tool-level Plans}

The desirability of using a certain tool can depend on many factors, including the forces required, the speed and accuracy with which it can be used, and the tools needed for previous and following operations. A planner based on this framework could easily output a list of possible tools to accomplish each task, to support such optimization in a later planning phases.

\section{Tool Interaction}

There might be two tools required in an operation at the same time that might interfere with each other. It is assumed that the feasibility of each tool can be determined independently of other tools used (either simultaneously or in sequence) in the same operation.

\section{Nonstandard Tools}

In some cases a special-purpose tool can be created when standard tools do no suffice, or a standard tool can be used in a nonstandard way (e.g., using a screwdriver to pound a nail).

\section{Design Changes}

The inability to use a standard tool often suggests that the design be changed to allow such access, but the framework does not go beyond this observation.

\section{References}

1. Proc. IEEE Intl. Symp. on Assembly and Task Planning, 1995.

2. D. Allen and T. R. Roose. Life cycle assessment and design for environment. In P.M. Eisenberger. Editor, Basic Research Needs for Environmentally Responsive Technologies of the Future: An Integrated Perspective of Academic, Industrial, and Government Researchers. Princeton Materials Institute, 1996.

3. G. Boothroyd, P. Dewhurst, and W. Knight. Product Design for Manufacture and Assembly. Marcel Dekker, 1994.

4. M. Brady, P. E. Agre, D. J. Braunegg, and J. H. Connell. The mechanic's mate. In T. O'Shea, editor, Advances in Artificial Intelligence, Elsevier, 1985.

5. D. S. Burks, M. D. Marks, and K. Ishii. Life cycle design for recyclability. In D. NavinChandra, editor, Green Engineering, Academic Materials Institute, 1996.

6. Y. Descotte and J.-C. Latombe. Making compromises among antagonist constraints in a planner. Artificial Intelligence, 27(2):183-217, 1985. 
7. A. Díaz-Calderón, D. Navin-Chandra, and P. K. Khosla. Measuring the difficulty of assembly tasks from tool access information. In Proc. IEEE Intl. Symp. on Assembly and Task Planning, pages 87-93, 1995.

8. R. Gadh, Z. Ashai, and K. Lee, Computer-aided design-for-disassembly; as applied to designfor-environment. Technical Report I_CARVE-1091, Dept. ME, Univ. of Wisconsin, 1994.

9. K. Green, E. Eggert, L. Stark, and K. Bowyer. Generic recognition of articulated objects through reasoning about potential function. CVGIP: Image Understanding, 62(2):177-93, 1995.

10. J. Henroiud and A. Bourjault. LEGA: a computer-aided generator of assembly plans. In [6], pages 191-215.

11. R. L. Hoffman. Automated assembly in a CSG domain. In Proc. IEEE Intl. Conf. On Robotics and Automation, pages 210-215, 1989.

12. L. S. Homen de Mello and S. Lee, editors. Task Sequence Planning for Robotic Assembly. $\mathrm{PhD}$. Thesis, Carnegie-Mellon Univ., 1989.

13. L. S. Homen de Mello and S. Lee, editors. Computer-Aided Mechanical Assembly Planning. Kluwer, 1991.

14. L. S. Homen de Mello and A. C. Sanderson. A correct and complete algorithm for the generation of mechanical assembly sequences. IEEE Trans. on Robotics and Automation, 7(2):228-240, 1991.

15. S. G. Kaufman, R. H. Wilson, R. E. Jones, T. L. Calton, and A. L. Ames. The Archimedes 2 mechanical assembly planning system. In Proc. IEEE Intl. Conf. on Robotics and Automation, pages 3361-8, 1996.

16. Y. Koga, K. Kondo, J. Kuffner, and J.-C. Latombe. Planning motions with intentions. In Proc. SIGGRAPH, pages 395-408, 1994.

17. D. J. Kriegman and J. Ponce. Computing exact aspect graphs of curved objects: Solids of revolution. Intl. J. of Computer Vision, 5(2):119-135, 1990.

18. J. M. Miller and R. L. Hoffman. Automatic assembly planning with fasteners. In Proc. IEEE Intl. Conf. On Robotics and Automation, pages 69-74, 1989.

19. R. Narang and G. Fisher. Development of a framework to automate process planning functions and to determine machining parameters. Intel. J. of Production Research, 31(8):1921-42, 1993.

20. D. Navin-Chandra. ReStar: A design tool for environmental recovery analysis. In Proc. Intl. Conf. On Engineering Design, 1993.

21. N. J. Nilsson. Principles of Artificial Intelligence. Springer-Verlag, 1980.

22. A. Requicha and J. Vandenbrande. Automatic process planning and part programming. Technical Report 217, Inst. for Robotics and Intelligent Systems, 1987.

23. A. Spyridi and A. Requicha. Accessibility analysis for the automatic inspection of mechanical parts by coordinate measuring machines. In Proc. IEEE Intl. Conf. on Robotics and Automation, pages 1284-1289, 1990. 
24. R. H. Wilson. Minimizing user queries in interactive assembly planning. IEEE Trans. On Robotics and Automation, 11(2):308-312, 1995.

25. R. H. Wilson and J.-C. Latombe. Geometric reasoning about mechanical assembly. Artificial Intelligence, 71(2):371-396, 1994.

26. J. D. Wolter. On the Automatic Generation of Plans for Mechanical Assembly. PhD thesis, Univ. of Michigan, 1988. 


\section{4}

\section{Automatic Planning for Manufacturing Generative Processes}

Generative process planning describes the methods process engineers use to modify manufacturing (or process) plans after a design is complete. A completed design may be the result from the introduction of a new product based on an old design, assembly upgrade, or modified product designs used for a family of similar products. Typically, an engineer designs an assembly and process plans are created capturing the manufacturing processes, including the assembly sequence, the methods used to put the piece parts together, the cost of the piece parts, labor costs, etc. When new products originate as a result of an upgrade, the geometry of parts may change, and/or additional components and subassemblies are added to or are omitted from the original design. As a result, process engineers are forced to create a "new" set of process plans. Often times, this is a tedious and time-consuming task, even if only a single component is involved in the upgrade. The task is further complicated by the fact that the process engineer is forced to manually generate these plans for each product upgrade. Assembly planners, including Archimedes $3.0^{\circ}$, can not automatically handle the upgrade modifications. To generate new assembly plans for the product upgrade, engineers have to manually re-specify the manufacturing plan selection criteria and re-run the planners. To remedy this problem, special-purpose routines have been added to the Archimedes $3.0^{\circ}$ planning algorithms and constraint framework. 
Section 4.1 provides an overview of the motivational factors for implementing automatic generative process planning techniques. Section 4.2 places the Archimedes $3.0^{\oplus}$ System in the context of generative process planning. Section 4.3 discusses the geometric issues associated with top-level assembly planning, and the special-purpose routines that were implemented within the Archimedes $3.0^{\odot}$ framework to solve those problems. Section 4.4 continues this discussion, but at the subassembly-level. Methods are presented for saving, restoring, and propagating the subassembly analyses for top-level assembly analysis. Section 4.5 describes the implementation of constraint rules enabling a user to automatically "reconcile" existing constraints applied to an older version of an assembly to a new version of an assembly. Finally, Section 5.6 concludes the Chapter and presents future research topics in this area.

\subsection{Background and Motivation}

The Archimedes $3.0^{\circ}$ System has been applied to hundreds of assemblies, ranging from automotive and aircraft to such things as designing assembly sequences for several weapon safety devices and for the B61. The B61 is an improved de-nuclearized bomb that was designed with a heavy reinforced nose cone that allowed it to penetrate the ground before detonation. It replaced the aging B53, which was difficult to maintain and to monitor for safety in storage. The scope of the modifications to the B61 requires exhaustive testing to certify the modified bomb's safety, functionality, and reliability. In an early experiment, Archimedes $3.0^{\circ}$ was applied to the B61 center-case for the B61 Alt 339 Retrofit Program. It was estimated that 2.5-3 person months were required to manually create training documentation for the retrofit operations using a commercial animation package.

In an effort to reduce the time, Archimedes $3.0^{\odot}$ was applied to the B61 center-case assembly. Unfortunately, the experiment showed that there were many assembly planning issues associated with CAD revisions that went beyond the disassembly issues discussed in the previous chapter. For instance, the first step required to apply Archimedes $3.0^{\odot}$ was to translate the Pro/ENGINEER $\otimes$ data to the ACIS format. Initially, the entire center-case assembly, containing 547-parts and represented by over $600 \mathrm{~K}$ facets, was selected for the Archimedes $3.0^{\odot}$ application; however, due to translation the problems a 303-part subassembly (345K facets) was exercised during the experiment. Effectively, the original design was modified by removing parts.

Archimedes $3.0^{\circ}$ was first applied to the original (larger) solid model to identify any inconsistencies in the CAD model. This allowed for the detection of critical design flaws to be caught early in the re-manufacturing phase and a reduction in scheduling and costs. Next, Archimedes $3.0^{\ominus}$ was used to test the feasibility of disassembly, checking for geometric accessibility for removal of parts. Since Archimedes $3.0^{\circ}$ plans only for straight-line motions, and this assembly contained a number of flexible parts, such as cables, that could not use straight-line assembly motions, the part-mating operations involving those parts were overrode. This was a long and tedious manual process. When it was decided to exercise the smaller assembly, these same tedious steps had to be repeated, almost identically to the first application, since Archimedes $3.0^{\ominus}$ can not reconcile the differences (part count, geometry, constraints and overrides) between the two assemblies automatically. 
This same problem was inherent in the application of Archimedes $3.0^{\circ}$ to the nose assembly (shown in Figure 5.1) at the Federal Manufacturing \& Technologies Facility at Allied Signal in Kansas City. In this case a new modification and proposed design of the B61 nose assembly incorporated new radar hardware and sophisticated structure elements to withstand high-shock environments. During hardware evaluation stages at Allied Signal, the system was used to determine re-manufacturability of the B61 nose assembly. Pro/ENGINEER ${ }^{8}$ parts were transferred to the Archimedes $3.0^{\circ}$ System for validation, assembly plans were evaluated, and assembly instructions and options were evaluated with process engineers. The nose assembly contains 88 parts described by $17.5 \mathrm{Mb}$ of ACIS $\otimes$ data (translated from Pro/ENGINEER $\otimes$ ) and approximately 110,000 facets.

Unlike the center-case assembly, the nose assembly went through several revisions before being finalized; however just like the center-case assembly application, for each revision the assembly planning steps had to be repeated (often duplicated). To complicate matters further, Archimedes $3.0^{\circ}$ had to be applied to each subassembly even if they were identical.

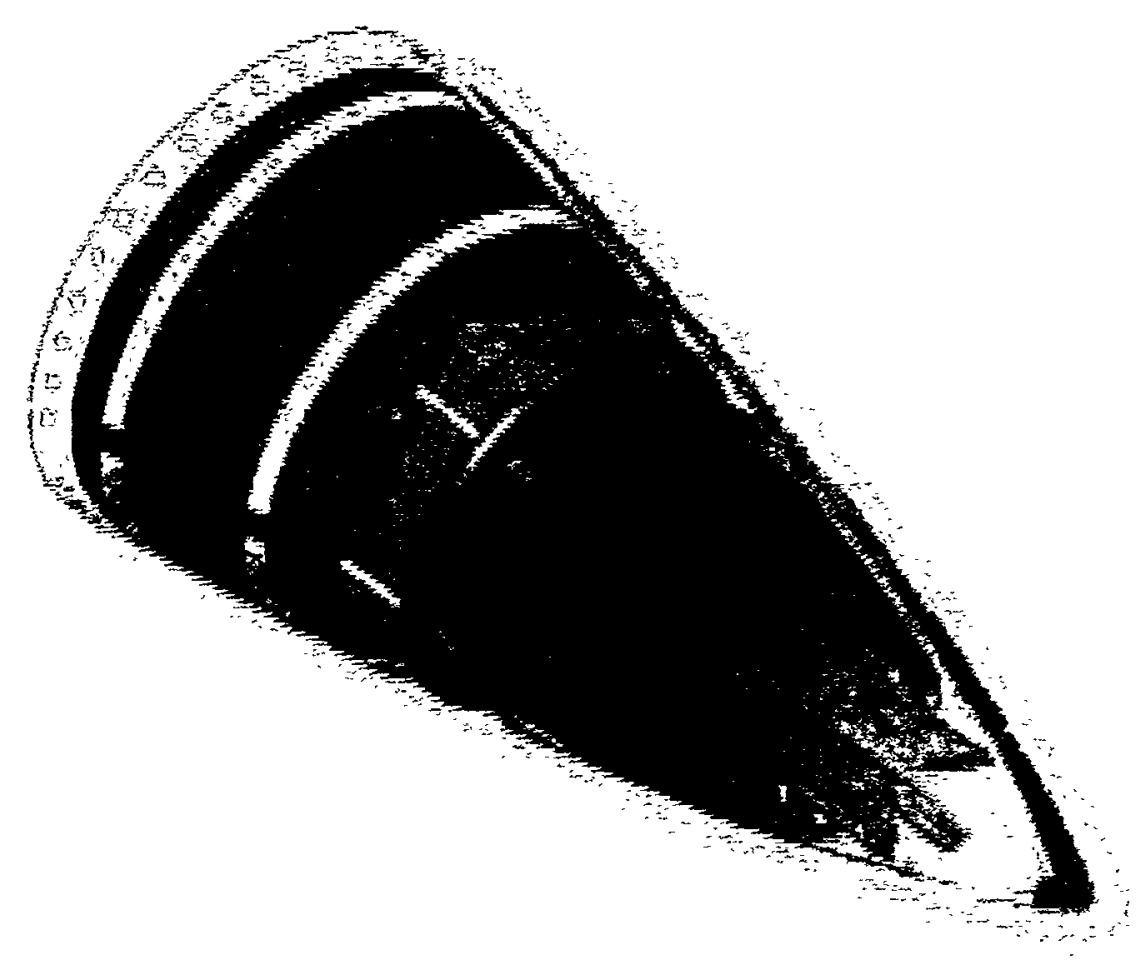

Figure 4.1. The B61 nose assembly. 


\subsection{Generative Process Planning Issues}

In the previous section four fundamental problems were identified during the application of Archimedes $3.0^{\oplus}$ to the B 61 .

1. Automatic planning with subassemblies: When assemblies are built by combining subassemblies, it is sometimes more practical to analyze the assembly at the subassembly level. That is, analyze each subassembly independently and then combine the analyses at the top-level (the assembly level). Archimedes $3.0^{\circ}$ plans for assembly at the top-level of the assembly and does not allow the propagation of information resulting from independent applications at the subassembly-level to the top-level.

2. Multiple identical subassemblies: If an assembly contains exact copies of the same subassembly and that subassembly requires any instantiation of constraints and overrides, the Archimedes $3.0^{\odot}$ user must instantiate them in each subassembly (duplicating his/her efforts on each of the identical subassemblies). This is because Archimedes $3.0^{\oplus}$ plans for assembly at the top-level of the assembly.

3. Assembly upgrade via re-design: An assembly may be modified by removing part(s), by changing the shape of the part(s), by adding parts(s), or by any combination of the three. Relating to the first two problems, is the inability of the Archimedes $3.0^{\oplus}$ System to automatically reconcile not only these modifications between design revisions, but also any constraints and overrides applied to the assembly for each generation.

4. Gaining access to selected parts for servicing ${ }^{2}$. This is the often associated with partial disassembly. Partial disassembly does not always proceed from or result in the removal of all of the parts in an assembly. For instance, a field upgrade may only require partial disassembly of a system to replace specified subassemblies. Archimedes $3.0^{\odot}$ does not plan for partial disassembly.

The problems identified above are not restricted to Archimedes $3.0^{\circ}$. They represent a fundamental class of problems inherent in all assembly planners and have plagued the manufacturing community for years. It is only recently, with the advancements in computer technology, that these problems have been brought to the forefront. With the exception of problem 4 , the remainder of this chapter is devoted to solving these problems. The underlying principles, as they relate to automatic assembly planning, are discussed, and solutions to each are provided. The solutions have been implemented as extensions to the Archimedes $3.0^{\circ}$. Solutions to problem 4 are provided in the next chapter.

${ }^{2}$ This issue is addressed in more detail in the next chapter. Archimedes $3.0^{\odot}$ was applied to the B61 tail-section for parachute inspection and servicing. 


\subsection{Re-design}

There are two fundamental issues associated with assembly design modification: geometry and function. For purposes of assembly planning only the geometric will be discussed. There are three geometry-related design modification principles for any given assembly. An assembly may be modified by removing part(s), by changing the shape of the part(s), by adding parts(s), or by any combination of the three.

In general, part removal is primarily motivated by a company's need to reduce the time it takes to put the product together. Obviously, the greater the number of parts, the higher the assembly costs. From an assembly standpoint, it is the simplest form of modification to deal with. Changing the geometry of a part is often the result of assembly facility and shop floor contingencies. For instance, some shapes are better suited for robotic assembly as opposed to manual assembly. The most difficult issue to address when planning assembly processes automatically for a product is the case where new parts are added to an existing assembly design.

Figure 4.2 shows an example assembly ${ }^{3}$ having gone through 3 design revisions. Initially, the assembly was comprised from 24 pieces: a base plate, two side plates, a top rail, and 20 various fastener piece parts (nuts, bolts, and washers). In an effort to reduce costs by reducing time spent assembling the product (i.e., reduce part count), several re-designs were made. In the first revision (R1), the fastening processes were addressed. As a result the fasteners were replaced with self-locking screws and inserted from the top. Hence, the results from the first revision reduced the part count to 8 . In the second revision (R2), the base plate and two side plates were re-designed into a single unit. With this modification, two new parts were incorporated. These are the clamp-like fasteners to hold the top rail in place. In the final revision (R3), the base is redesigned with tabs to hold the top rail, and the clamp-like fasteners are removed. Thus, the product has been reduced to 2 components.

In this simple example, all three re-design principles were implemented. This is true for almost all product upgrades. The first revision was a result of removing parts and adding new ones. In the second revision, part geometry changed and new parts were added. In the third revision, part geometry changed and parts were eliminated. The fundamental problem relating these re-design principles to automatic assembly planning is the inability of the planners to automatically recognize the changes between generations.

To address the first, the removal of parts, a geometric override was added to the Archimedes $3.0^{\circ}$ override architecture that removes all associations of that part with others (e.g., part contacts, overrides, and constraints) and effectively hides the part from the user's view. In Archimedes $3.0^{\oplus}$, routines to save and restore assembly plans, assembly constraints, and geometric overrides are implemented at the top-level assembly. This allows a user to analyze an assembly at the toplevel and save all of the analysis information. When the system is applied to the same assembly at a later time or to different generations of that assembly, the information may be invoked by restoring the files.

\footnotetext{
${ }^{3}$ This assembly was borrowed from [Boothroyd, G., "Assembly automation and product design", Marcel Dekker, New York, 1991, p. 14.] for illustrative purposes only. Hypothetical processes are used to illustrate the principles of re-design as they relate to automatic assembly planning.
} 


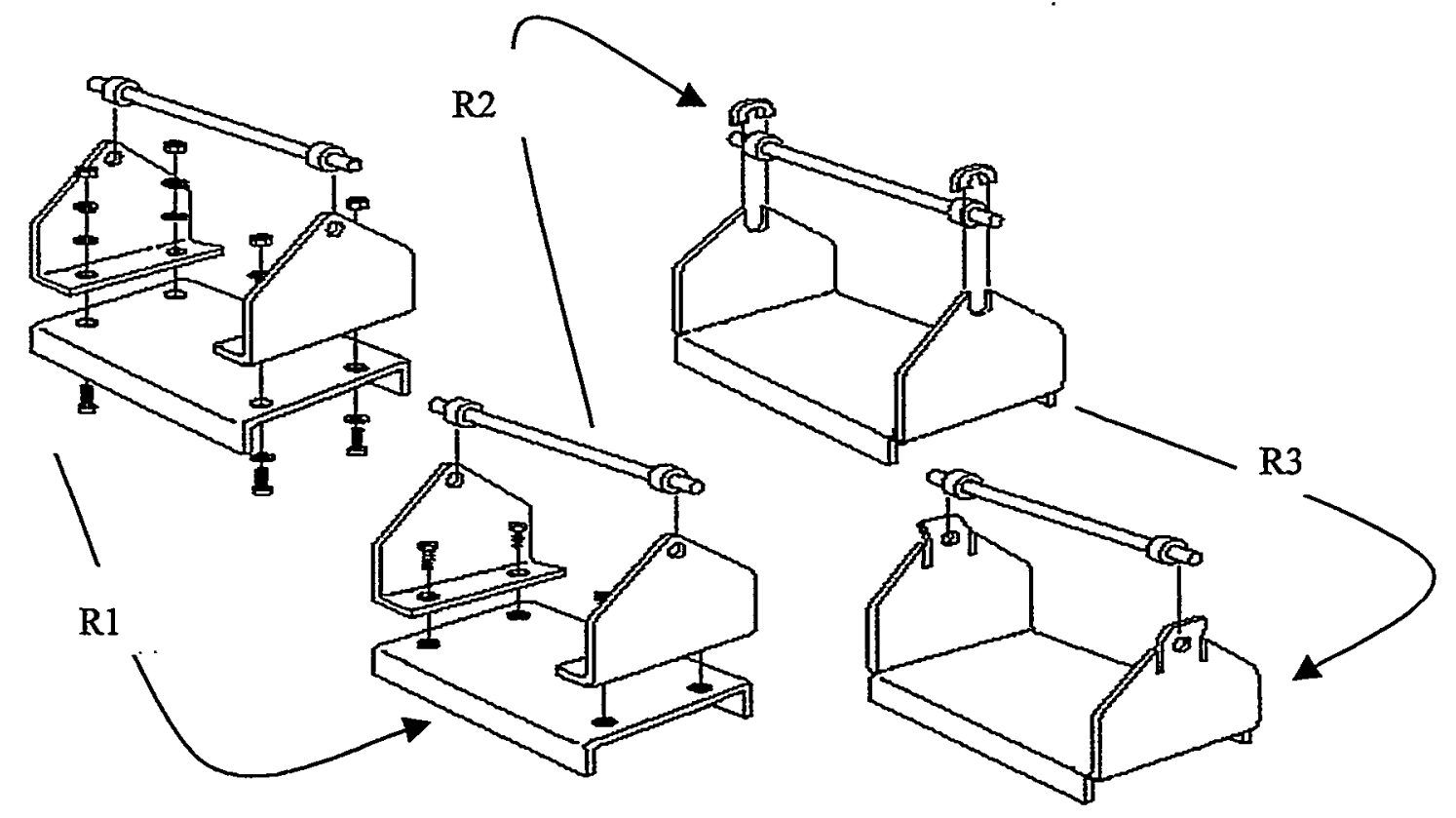

Figure 4.2. Example of the effects of re-design.

When the user loads in the assembly, the constraints and override files are automatically loaded. The assembly is represented by data bit-vector. The length of vector corresponds to the number of parts in the assembly. In all constraints and overrides, a " 0 " in a particular bit means one thing about a part and a "l" means something else, depending the type of constraint or override that is implemented. In this case, a " 0 " in the bit-vector notifies the system that that particular part is no longer in the assembly. While the part is still present in the assembly tree (i.e., the length of the bit-vectors for all constraints and overrides is constant), for all intense purposes it has been removed.

To address the second, changing component geometry, the Archimedes $3.0^{\circ}$ contact analysis routines automatically check contacts between parts. If the re-design changes the contacts between the parts, the user is automatically informed and is given the opportunity to address the issue. The same holds true for constraints and overrides. If a previously defined constraint or override is now in conflict, the user is automatically informed and is given an opportunity to address the issue(s).

The third issue, the addition of parts, is the most difficult issue. Because Archimedes $3.0^{\odot}$ plans for assemblies at the top-level and the length of data bit-vector representing the number of parts at the top-level is fixed, the Archimedes $3.0^{\oplus}$ System can not plan for assemblies upgrades at the top-level when the part count increases. This is a major research area on its own, and attention to solving this problem should be given to future work in this area. 


\subsection{Planning with Subassemblies}

This section deals with the generative process planning problems, 1 and 2, discussed in Section 4.2.

1. Analyzing each subassembly independently and then combining the analyses at the top-level (the assembly level).

2. Multiple identical subassemblies: If an assembly contains exact copies of the same subassembly and that subassembly requires any instantiation of constraints and overrides, the Archimedes $3.0^{\circ}$ user must instantiate them in each subassembly (duplicating his/her efforts on each of the identical subassemblies).

It is pointed out in the previous section that Archimedes $3.0^{\oplus}$ plans for assembly at the toplevel of the assembly and does not allow the propagation of information resulting from independent applications at the subassembly-level to the top-level. The first step to solving these problems was to incorporate save and restore routines for the constraints and overrides resulting from the application of Archimedes $3.0^{\circ}$ to the subassemblies, which would automatically load when Archimedes $3.0^{\Theta}$ was applied at the top level of the assembly. The underlying problem with this approach is how to resolve conflicts between the constraints and overrides when they are propagated to the top. Section 4.5 addresses the conflict resolution issues. Here, the methodologies for automatically propagating the information generated at the subassembly-level to the top-level are presented.

\subsubsection{Automatic Propagation}

File-restoration subroutines were incorporated into Archimedes $3.0^{\circ}$ to automatically load and propagate the constraints and overrides from the subassembly files to the top-level assembly. When Archimedes $3.0^{\odot}$ is applied at the subassembly-level, constraints and overrides are stored under the default name of the subassembly (e.g., subassembly-name.constraints and subassemblyname.overrides). When loading an assembly (the base assembly or top-level assembly), the subassembly constraints are automatically loaded using the subassembly-name.constraints default file. For the constraint restoration subroutine that restores two subassembly sets, bits in the data vector are set as follows for: (visible - 0 and group -1). For the constraint restoration subroutine that restores three subassembly sets, bits in the data vector are set as follows for: (visible - 1 , secondgroup - 0 , and group - 0 ). For each subassembly file restoration, the number-of-parts bit for the full assembly set is equal to the number of parts in the subassembly. The restoration algorithm changes the subassembly's portion of the assembly data vector to be that read from the subassembly file. Any parts in the vector not belonging to the subassembly are set to 0 . The algorithm changes the number-of-parts bit to equal the number of bits set in the data vector.

When loading an assembly, the subassembly overrides are also automatically loaded using the subassembly-name.overrides default file. The subassembly overrides are loaded with a new override class feature, called IsTopLevel, set 0 (or false), to indicate that they were loaded from the subassembly's overrides file, not from the base assembly's overrides file. The assembly overrides are created with IsTopLevel set to 1 (true). Only top-level overrides are saved for an assembly. Conflicting overrides made at the base assembly level are intended to take precedence over overrides made locally to the subassembly. 


\subsubsection{Demonstration of Propagation Effects}

To help illustrate the propagation of design modifications imposed at the subassembly-level for later use in planning at the top-level, conceptual designs of two assemblies are provided below.

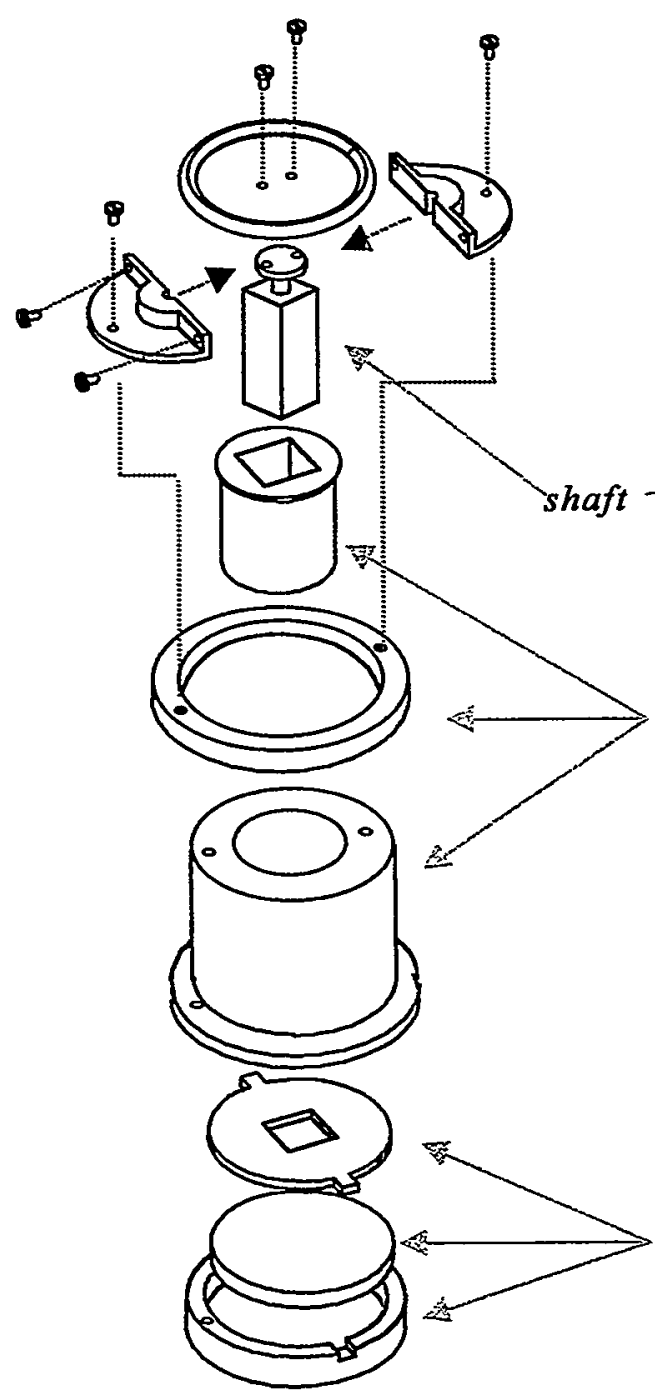

Assembly $A$

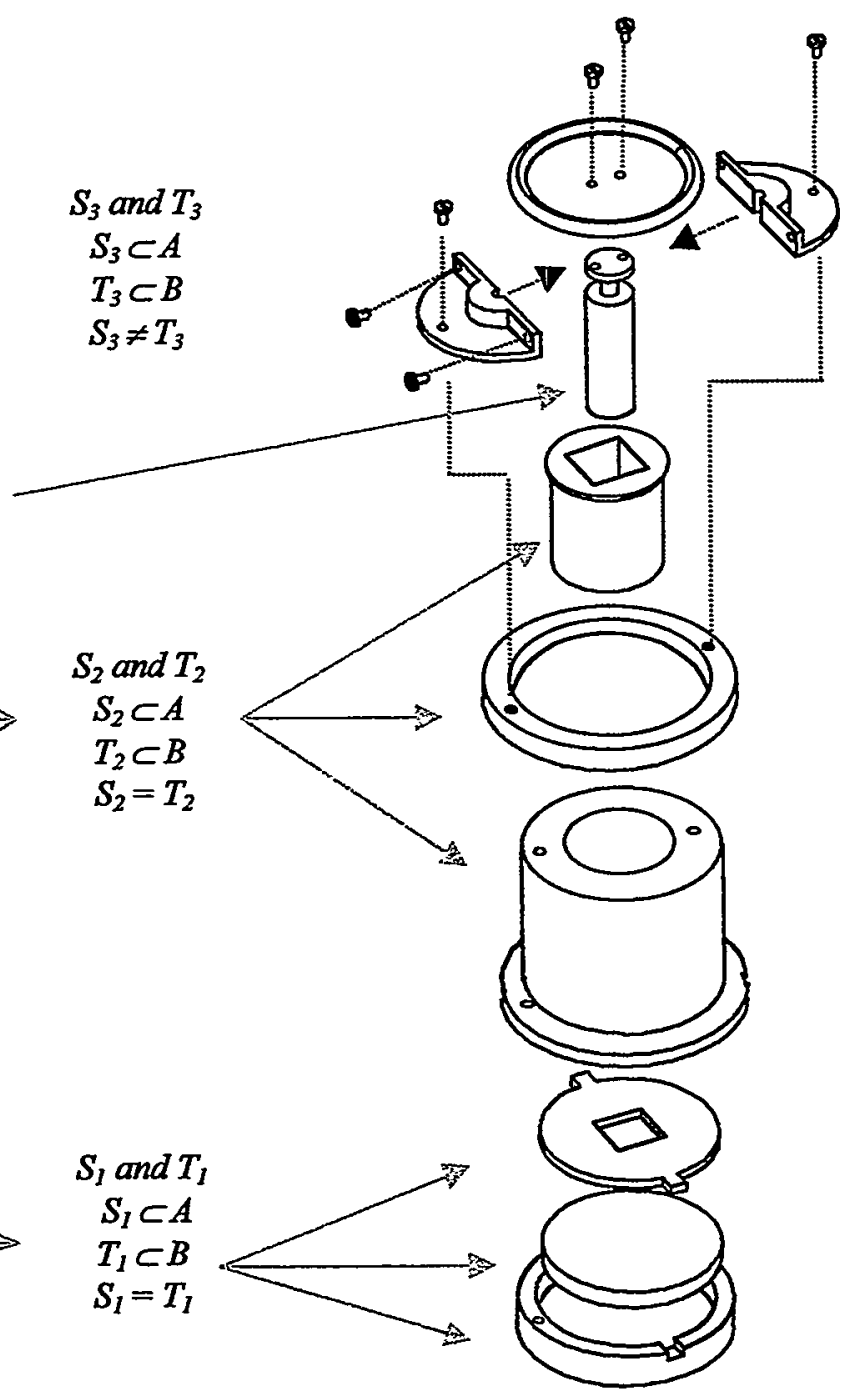

Assembly $B$

Figure 4.3. Demonstration of propagation effects for planning with subassemblies. Exploded diagrams for Assembly $A$ and Assembly $B$. 
Figure 4.4 shows exploded diagrams of two very similar assemblies, $A$ and $B$. The only difference between the two is the shape of the shaft in each. Assemblies $A$ and $B$ are made from three subassemblies and 6 fasteners. Viewing the diagram from the bottom up, the first three parts make up the first set of subassemblies, $S_{1}$ and $T_{1}, S_{1} \subset A, T_{1} \subset B, S_{1}=T_{1}$. The next three parts make up the second set of subassemblies, $S_{2}$ and $T_{2}, S_{2} \subset A, T_{2} \subset B, S_{2}=T_{2}$. All the remaining parts (with the exception of the fasteners) make up the third set of subassemblies, $S_{3}$ and $T_{3}, S_{3} \subset A, T_{3} \subset B, S_{3} \neq T_{3}$.

Suppose that $A$ ssembly $B$ is modified at the top-level by changing the shape of a part in $T_{2}$ as shown in Figure 4.4. Then the change only affects $B$. However, if the $B$ is modified at the subassembly-level (at $T_{2}$ ) then $A$ is no longer feasible.
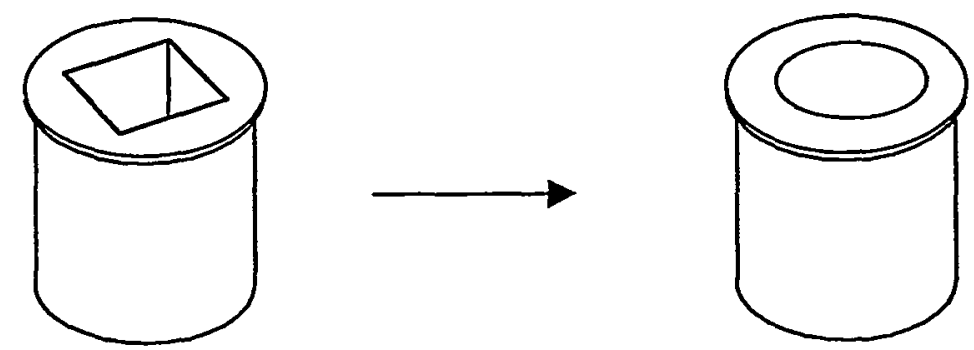

Figure 4.4. Design modification for Subassembly $T_{2}$.

On the other hand, suppose that Assembly $A$ is modified by lengthening the shaft and by cutting a rectangular hole in the plate to slide it into (see Figure 4.5). Then, in this case, the change does not affect Assembly $B$ at any level of planning.
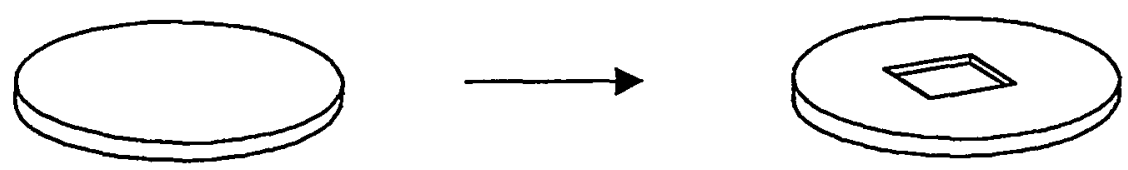

Figure 4.5. Design modification for Subassembly $S_{l}$. 


\subsection{Conflict Resolution between Generative Plans}

Rules were incorporated in Archimedes $3.0^{\Theta}$ to resolve conflicts between top-level and subassembly-level constraints and overrides (when restoring, adding, editing, or activating) at the top-level of the assembly. This section describes the implementation of the constraint and override rules enabling the Archimedes $3.0^{\odot}$ System to automatically "reconcile" existing constraints applied to an older version of an assembly to a new version of an assembly.

\subsubsection{Constraints}

Based on the various constraint and intended purposes, four different methodologies were developed for the implementing the rules. A list of all constraints implemented in Archimedes 3.0 is provided in Chapter 1,Table 1.1. Table 4.1 lists constraint types that have been added to the Archimedes $3.0^{\odot}$ for life cycle assembly processes. In defining the methodologies, the term current constraint refers to the constraint that is being added, edited, or activated. The term existing constraint refers to the constraint that is in conflict with the current constraint.

The methodologies include:

1. Methods that suspend the existing constraint on conflict give top-level constraints precedence over subassembly constraints during restoration, and new additions or edit changes precedence over existing. The former happens because top-level constraints are restored last. (See Table 4.2.)

2. Methods that suspend the current constraint on conflict give subassembly-level constraints precedence over top-level constraints during restoration, and existing constraints take precedence over new additions or edit changes. The former happens because top-level constraints are restored last. In these cases it is assumed that the designer of the subassembly "knows" best. (See Table 4.3).

3. Methods that union constraints are shown in Table 4.4. Only one constraint of that type to be active.

4. Methods that do no conflict resolution, implement the current constraint addition or change regardless of any existing constraints. These constraints are listed in Table 4.5. 
Table 4.1. Additional constraints not provided in Chapter 1. Those marked with a are additional constraints required for life cycle assembly process planning.

\begin{tabular}{|l|l|l|}
\hline \multicolumn{1}{|c|}{ Constraint Name } & \multicolumn{1}{|c|}{ Purpose } & Scope \\
\hline $\begin{array}{l}\text { MIN_SIMUL_ } \\
\text { LIAISONS }\end{array}$ & $\begin{array}{l}\text { Minimize the use of simultaneous liaison creation. In } \\
\text { some contexts, actions are awkward when higher } \\
\text { numbers of liaisons are being established by the } \\
\text { action }\end{array}$ & strategic \\
\hline MIN_REORIENT & $\begin{array}{l}\text { Minimize the number of assembly orientations in the } \\
\text { plan. }\end{array}$ & strategic \\
\hline REQ_SUCCESS_PART & $\begin{array}{l}\text { Allows the user to specify a part or collection of parts } \\
\text { that must be removed from an assembled product. } \\
\text { This is especially useful for servicing, repair, and } \\
\text { upgrade operations. }\end{array}$ & strategic \\
\hline $\begin{array}{l}\text { REQ_SUBSEQUENCE_ } \\
\text { PARTS }\end{array}$ & $\begin{array}{l}\text { Allows the user to specify the order in which a } \\
\text { subsequence of parts is assembled. This useful for } \\
\text { controlling the planning algorithms in circumstances } \\
\text { where a specific ordering is already known in } \\
\text { advance. }\end{array}$ & strategic \\
\hline REQRECONFIGURE & $\begin{array}{l}\text { Allows the user to specify reconfigurations of the } \\
\text { assembly so that parts can be moved to and from their } \\
\text { final configurations. }\end{array}$ & strategic \\
\hline
\end{tabular}


Table 4.2. List of constraints and rules that give top-level constraints precedence over subassembly constraints. Those marked with * are constraints not available in Archimedes $3.0^{\circ}$.

\begin{tabular}{|l|l|}
\hline \multicolumn{1}{|c|}{ Constraint Name } & \multicolumn{1}{c|}{ Rules } \\
\hline REQ_ORDER_LIAISON & $\begin{array}{l}\text { For two REQ_ORDER_LIAISON constraints, if current group } \\
\text { intersects with existing second group, and the current second group } \\
\text { intersections with existing group, then suspend the existing constraint. }\end{array}$ \\
\hline REQ_ORDER_PART & $\begin{array}{l}\text { For two REQ_ORDER_PART constraints, if the current group } \\
\text { intersects with the existing second group, and the current second } \\
\text { group intersects with existing group, then suspend the existing } \\
\text { constraint. }\end{array}$ \\
\hline REQ_PATHS_AXIAL & $\begin{array}{l}\text { If two REQ_PATHS_AXIAL constraints intersect and their required } \\
\text { paths are not equal, then suspend the existing constraint. }\end{array}$ \\
\hline REQ_STACK & $\begin{array}{l}\text { If two REQ_STACK constraints intersect and their required } \\
\text { trajectories are not equal, suspend the existing constraint. }\end{array}$ \\
\hline REQ_SUBSEQ & $\begin{array}{l}\text { If two REQ_SUBSEQ constraints are the same type (assembly or } \\
\text { disassembly) and their groups intersect, then suspend the existing } \\
\text { constraint. }\end{array}$ \\
\hline REQ TOOL & $\begin{array}{l}\text { If two REQ TOOL constraints primary parts are the same, suspend } \\
\text { the existing constraint. }\end{array}$ \\
\hline $\begin{array}{l}\text { REQ_SUBSEQUENCE_ } \\
\text { PARTS* }\end{array}$ & $\begin{array}{l}\text { If two REQ_SUBSEQUENCE_PARTS groups intersect, then } \\
\text { suspend the existing constraint. }\end{array}$ \\
\hline
\end{tabular}

Table 4.3. List of constraints and rules that give subassembly-level constraints precedence over top-level constraints. Those marked with * are constraints not available in Archimedes $3.0^{\circ}$.

\begin{tabular}{|l|l|}
\hline \multicolumn{1}{|c|}{ Constraint Name } & \multicolumn{1}{c|}{ Rules } \\
\hline REQ SUBASSY & $\begin{array}{l}\text { If two REQ SUBASSY constraints intersect, but neither is a subset } \\
\text { of the other, then suspend the current constraint. If the current } \\
\text { REQ_SUBASSY intersects with an existing } \\
\text { REQ_SUBASSY_WHOLE, but neither is a subset of the other, then } \\
\text { suspend the current constraint. }\end{array}$ \\
\hline $\begin{array}{l}\text { REQ SUBASSY_ } \\
\text { WHOLE }\end{array}$ & $\begin{array}{l}\text { If two REQ_SUBASSY_WHOLE constraints intersect, but neither is } \\
\text { a subset of the other, then suspend the current constraint. If current } \\
\text { REQ_SUBASSY_WHOLE intersects with an existing } \\
\text { REQ_SUBASSY, but neither is a subset of the other, then suspend } \\
\text { the current constraint. }\end{array}$ \\
\hline
\end{tabular}


Table 4.4. List of constraints and rules that union top-level and subassembly constraints. Those marked with * are constraints not available in Archimedes $3.0^{\circ}$.

\begin{tabular}{|l|l|}
\hline \multicolumn{1}{|c|}{ Constraint Name } & \multicolumn{1}{c|}{ Rules } \\
\hline REQ ORDER_FIRST & $\begin{array}{l}\text { If the current REQ ORDER_FIRST intersects with an existing } \\
\text { REQ_ORDER_FIRST, suspend the existing constraint. Union } \\
\text { current constraint with any existing REQ_ORDER_FIRST } \\
\text { constraints and delete the existing constraints. }\end{array}$ \\
\hline REQ_ORDER_LAST & $\begin{array}{l}\text { If the current REQ_ORDER_LAST constraint intersects with an } \\
\text { existing REQ ORDER_FIRST constraint, suspend the existing } \\
\text { constraint. Union the current constraint with any existing } \\
\text { REQ ORDER_LAST constraints and delete the existing constraints. }\end{array}$ \\
\hline $\begin{array}{l}\text { REQ SUCCESS_ } \\
\text { PART* }\end{array}$ & $\begin{array}{l}\text { Union current constraint with any existing REQ_SUCCESS_PART } \\
\text { constraints and delete the existing constraints. }\end{array}$ \\
\hline
\end{tabular}

Table 4.5. List of constraints that give top-level precedence and have no conflicts. Those marked with * are constraints not in Archimedes 3.0 .

\begin{tabular}{|l|l|}
\hline \multicolumn{1}{|c|}{ Constraint Name } & Rules \\
\hline REQ LINEAR_PARTS & None. \\
\hline PRH_STATE & None. \\
\hline PRH_SUBASSY & None. \\
\hline REQ_CLUSTER & None. \\
\hline REQ_FASTENER & None. \\
\hline $\begin{array}{l}\text { REQ LINEAR_ } \\
\text { CLUSTER }\end{array}$ & None. \\
\hline REQ_PART_SPECIAL & None. \\
\hline REQ_STAT & None. \\
\hline $\begin{array}{l}\text { MIN_SIMUL_ } \\
\text { LIAISONS* }\end{array}$ & None. \\
\hline MIN_REORIENT* & None. \\
\hline
\end{tabular}




\subsubsection{Overrides}

Rules for resolving conflicts between subassembly overrides and top-level overrides are much simpler than the constraints. When applying the system to the assembly at the top-level, overrides for the subassemblies (parsing the subassembly tree bottom-first, deleting similar overrides at it goes) are automatically loaded. The system then loads the overrides for top-level assembly (deleting similar overrides, in this case any non-top-level overrides of same type for the same part), if any exist. On restoring all top-level overrides from a file, the system removes all top-level overrides and then loads in new top-level overrides (deleting similar overrides).

\subsection{Conclusions and Future Work}

To the author's knowledge no automatic assembly planner has ever compensated for automatic planning for generative processes. The methodologies presented and implemented in this chapter are natural progressions towards fully automating generative process planning.

The system has been tested on numerous assemblies and has shown significant increases in efficiencies in planning for assembly upgrades. However, it is difficult to obtain precise measures of efficiency since there many variables affecting the process (e.g., the user of the system, the size of the assembly, the number of initial constraints and overrides to produce a feasible process plan, etc.).

Future work needs to be directed towards the development of algorithms to fully automate the propagation of the assembly constraints and overrides when parts are added to an assembly. 


\section{Automatic Planning for Partial Disassembly}

As in initial assembly, the product design and known process constraints are inputs to creating such plans. But for lifecycle assembly planning, the goal of the process is often different. Examples are replacement of particular subassemblies, or least-cost dismantlement and disposal. Furthermore, the disassembly and assembly processes used to meet these goals have important differences from initial manufacturing. In planning for and optimizing lifecycle disassembly sequences, there are substantial technical issues that require fundamental rethinking of the procedural representation mentioned above. That which is addressed in this chapter is partial assembly (or disassembly). In a partial disassembly process, assembly and disassembly does not always proceed from or result in individual parts. For instance, a service-oriented task may require only partially disassembly of a component, and then subassemblies are placed in it.

In the sections that follow, automatic planning for partial disassembly is discussed. Section 5.1 covers the inclusion of a newly implemented constraint, REQ SUCCESS_PART(s), into the Archimedes $3.0^{\oplus}$ System to accommodate for service-oriented assembly processes. The instantiation of a REQ SUCCESS_PART(s) constraint allows a user to specify a part or collection of parts that must be removed from an assembled product. This forces the planner to stop when none of the desired parts (as specified in the constraint) are left in the assembly. 
Section 5.2 further describes a computer algorithm and its implementation that optimizes the search strategy for the REQ SUCCESS_PART(s) constraint. This search strategy uses a hillclimbing technique of the standard $A^{*}$ search to repeatedly probe the subassembly tree, looking for better (less costly) disassembly sequences. An additional algorithm, referred to as the shortening algorithm, is also described. The purpose of the shortening algorithm is to shorten the initial sequence by eliminating unnecessary removal of parts not specified in the REQ SUCCESS_PART(s) constraint set. Finally, Section 5.3 concludes the chapter with an example application.

\subsection{Service-oriented Assembly Planning}

As a first approach to service-oriented part removal, the Archimedes $3.0^{\odot}$ disassembly planner was employed essentially as-is, by specifying a constraint, which is referred to as REQ SUCCESS_PART(s), which essentially cuts the planner off when none of the desired parts are left in a subassembly. In general, this does not produce very satisfactory disassembly plans, as the first disassembly sequence produced by the planner typically contains large numbers of unnecessary operations, which have nothing to do with getting at the desired parts.

As a next step, a more general search-optimization strategy was incorporated into Archimedes $3.0^{\oplus}$. This strategy uses a hill-climbing variety of the standard $\mathrm{A}^{*}$ search to repeatedly probe the subassembly tree, looking for better (less costly) disassembly sequences. Running with a REQ SUCCESS_PART constraint, and the optimizer enabled, causes Archimedes $3.0^{\circ}$ to iteratively run the disassembly planner, with an aim toward finding an "optimal" disassembly sequence. The debilitating drawback to this approach is that the size of the subassembly tree makes it impossible to find optimal solutions in reasonable time, for assemblies with more than about 20 parts. The nature of the optimization scheme is such that the algorithm will run until the cost of the best disassembly sequence found so far is equal to the lower bound on the cost of the best disassembly sequence possible. The problem is that if the base assembly admits a few hundred possible disassembly steps, the execution time required to find the best possible disassembly sequence is prohibitive. This demonstrates that even the best possible sequence is at least two operations deep and requires the exploration of tens of thousands of disassembly sequences. Finding a "best" disassembly sequence can also take a prohibitively long time. "Best means that a person can analyze an assembly sequence and say with certainty that a particular sequence is clearly the best possible. Archimedes $3.0^{\circ}$ may still be unable, using the generalpurpose optimizer, to find that sequence, let alone say that no other sequence is better.

\subsection{An Optimizing Search Algorithm for Assembly Sequences}

One of the reasons that the general-purpose optimizer does not provide adequate support for service-oriented part removal is that it is not capable of eliminating, in an efficient manner, what humans would think of as "obviously unnecessary" part removals from the disassembly sequence. For example, consider the partial disassembly sequence shown in Figure 5.1; the task is to remove the part labeled B from the assembly for service or replacement. It is obvious that the 
correct disassembly sequence is to remove the screws labeled $\mathrm{F}$ and $\mathrm{G}$ from the assembly and then to remove the cover plate, D. Because Archimedes $3.0^{\Theta}$ has no way of knowing, a priori, that removing screws $\mathrm{H}$ and $\mathrm{I}$ will not help in gaining access to part $\mathrm{B}$, it might return the sequence shown in the figure; removal of parts I, H, F, G, D, and then $B$. In this case the number of parts is small enough that the optimizer would quickly discover the sequence $\{F, G, D, B\}$, and also quickly discover that no cheaper sequence would achieve the removal of part B. But if the assembly had, say, 40 or 50 parts, Archimedes $3.0^{\oplus}$ might run for an arbitrarily long time before discovering the sequence $\{F, G, D, B\}$, and would run even longer before proving to its own satisfaction that a cheaper sequence did not exist.

In order to improve Archimedes $3.0^{\Theta}$, ability to find sequences that do not contain "obviously unnecessary" part removals, a specific module that operates on existing plans to improve their efficiency was implemented. The planner invokes two steps when a REQ SUCCESS_PART constraint is declared. First, the standard disassembly planner is used to provide an initial sequence $P$ that enables the removal of the desired part (in this case, $B$ ).

Second, a subroutine (hereafter referred to as the "shortening algorithm") is invoked which attempts to shorten $P$ by eliminating removal operations from it. This is accomplished by attempting to indefinitely defer specific removal operations. For the disassembly sequence shown in Figure 1, \{I, H, F, G, D, B \}, the planner attempts to determine if, instead of removing part I first, part $\mathrm{H}$ could be the first part removed. Since this is possible, the planner goes on to consider the sequence $\{H, I, F, G, D, B\}$. This sequence has all of the same operations, and the same cost, but has deferred the removal of part I for one operation. The planner then tries to defer I's removal by another step: $\{H, F, I, G, D, B\}$. It is successful, so it goes on to try $\{H, F$, G, I, D, B $\},\{H, F, G, D, I, B\}$, and finally $\{H, F, G, D, B, I\}$. For this final disassembly sequence, the planner realizes that it doesn't need to remove part $I$, having already removed part $B$, and so it returns the plan $\{H, F, G, D, B\}$, which is cheaper by the cost of removing part I.

If the planner is not successful in deferring the removal of the first part indefinitely, it goes on to try again with the second part, then the third part, and so on, until it reaches the end of the plan. If it never succeeds, then it simply returns the original plan. If, on the other hand, it does succeed in deferring the removal of any particular part indefinitely then it stops. The planner then recomputes the "best plan", and repeats, until it fails to indefinitely defer the cost of any part removal. In this example, after specifically generating the removal operations needed to provide the disassembly sequence $\{H, F, G, D, B\}$, the planner then goes on to try to defer the removal of part $H$, generating, in order, the sequences $\{F, H, G, D, B\},\{F, G, H, D, B\},\{F, G, D, H, B\}$, and finally $\{F, G, D, B, H\}$, which is truncated to $\{F, G, D, B\}$, which is, in this case, the optimal solution. 

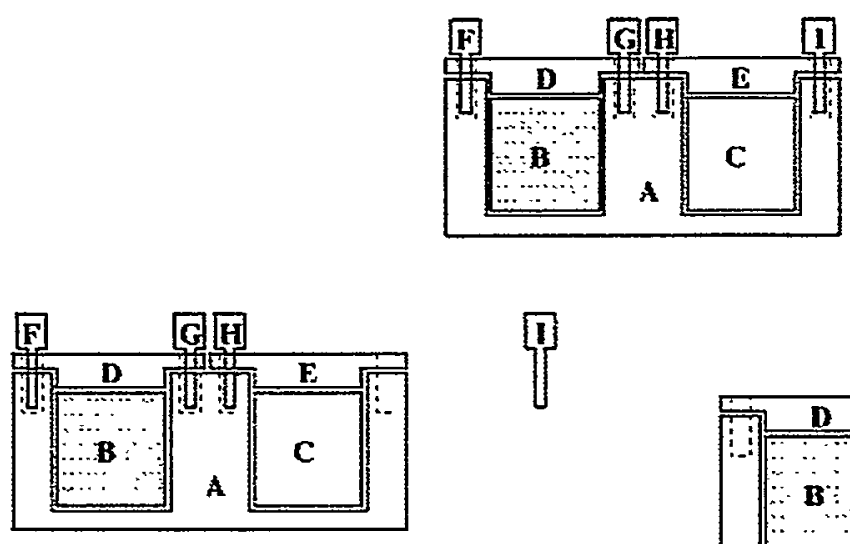

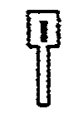
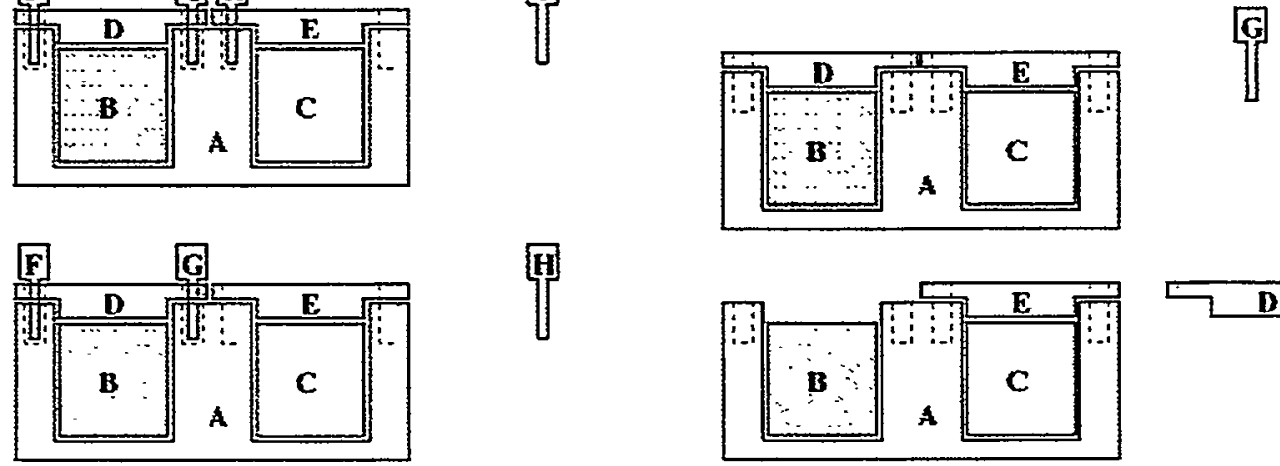

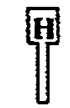
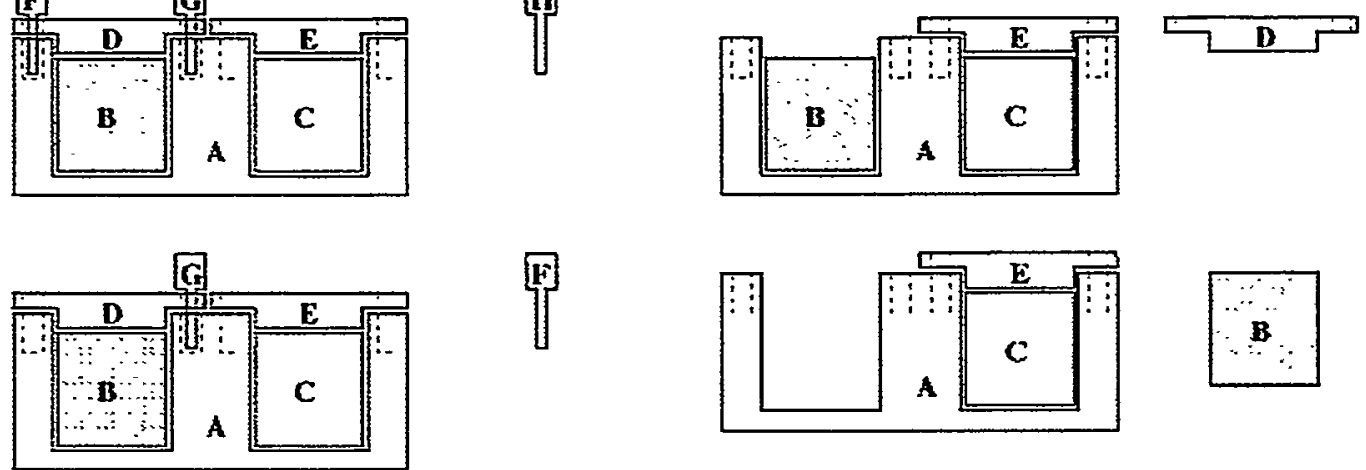

EF
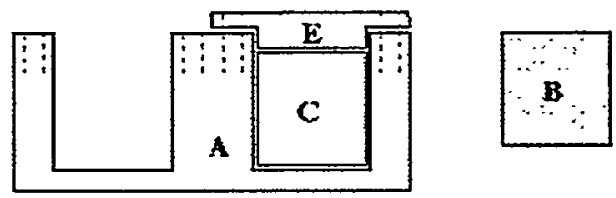

Figure 5.1. Example of an assembly from which a specific part is to be removed.

It must be stressed that this additional algorithm does not guarantee convergence of the optimized search for a cheapest disassembly sequence. This is true for two reasons. First, this algorithm does not provide any information about minimum possible costs. In this case, if there were 40 or 50 parts in the assembly, the algorithm would do nothing toward proving that there was not a cheaper sequence. If cost and length of the disassembly sequence are considered to be identical for the purposes of illustration, then this algorithm would do nothing toward showing that there does not exist a length 3 disassembly sequence terminating in the removal of part B from the assembly in Figure 5.1. 


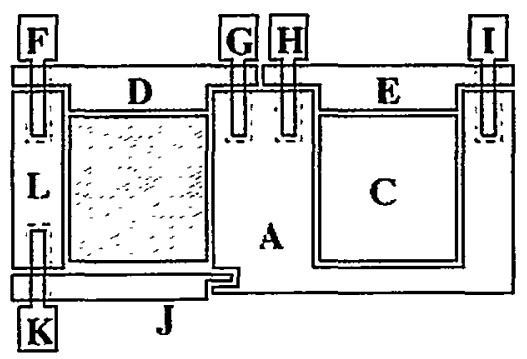

Figure 5.2. Example of assembly where the shortening algorithm is not guaranteed to find an optimally short disassembly sequence.

The second reason that the results of this algorithm are not guaranteed is that the effects of this algorithm are only visited upon plans found by the existing disassembly planner. Suppose for example in Figure 5.2, that part B was held within its cavity in part A, not only by the plate $D$, but also by a plate $J$, which came into the bottom of B's cavity (rather than the bottom of the cavity being a solid part of $\mathrm{A}$ ), but was held in place only by a single screw, $\mathrm{K}$. Then the optimal removal sequence would be $\{\mathrm{K}, \mathrm{J}, \mathrm{B}\}$. Until the planner found a sequence containing $\{\mathrm{K}, \mathrm{J}, \mathrm{B}\}$ as a subsequence, the shortening algorithm would not be able to produce $\{K, J, B\}$ as an alternative.

However, in spite of these limitations, in practice the shortening algorithm does provide an effective way to find a lowest-cost servicing disassembly plan. The user can specify the set of parts for the service operation, enable the optimizer, start the planner, and let it run until the cost of the best plan so far stays the same for several iterations. At that point the plan will, with high probability, be the one desired. If not, the user can either rerun the planner for a longer duration, or add constraints to help the planner find the minimum cost service plan.

\subsection{Experiments}

The Archimedes $3.0^{\odot}$ planner, extended with the constraint system (see Chapter 1 ) to a number of actual assemblies from sources in government and industry. To date, the newly integrated shortening algorithm has only been implemented in test case scenarios.

The $\mathrm{B} 61$ is an improved de-nuclearized bomb, which was designed with a heavy reinforced nose-cone that allowed it to penetrate the ground before detonation. It replaced the aging B53, which was difficult to maintain and to monitor for safety in storage. The scope of the modifications to the B61 require exhaustive testing to certify the modified bomb's safety, functionality, and reliability.

With the shortening algorithm, it is possible to generate fewest-removed-parts-disassembly plans for easy access to parts that require servicing or replacement on a regular basis, such as the parachute shown in the B61 tail-section in Figure 5.3. As part of routine maintenance, the parachute is regularly removed from the tail-section with a flared cone, which changed the 
appearance of the weapon somewhat in the rear, where the fins existed before to allow the bomb to spin in a certain way. In Figure 5.3, the picture on the left depicts the tail-section subassembly in its assembled configuration. On the right is a picture showing the tail-section with the parachute partially removed after the shortening was applied.

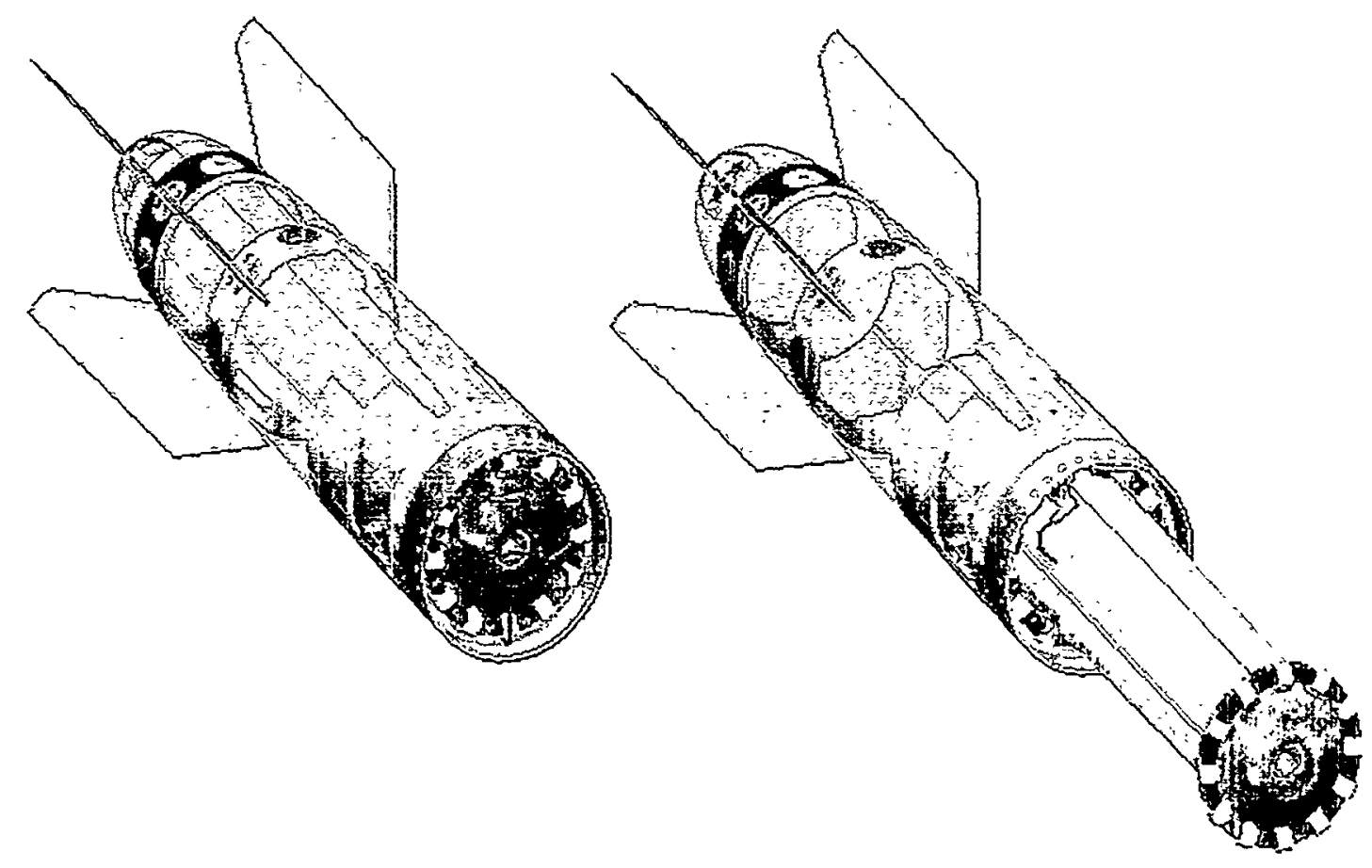

Figure 5.3. The tail-section subassembly of the B61 Bomb.

The tail-section subassembly contains 258 parts described by $17.5 \mathrm{Mb}$ of ACIS $\circledast$ data (translated from Pro/ENGINEER@) and slightly over a million facets. Archimedes was first applied to the solid model to identify any inconsistencies in the CAD model. This allowed for the 
detection of critical design flaws to be caught early in the re-manufacturing phase (servicing) and a reduction in scheduling and costs. Next, Archimedes was used to test the feasibility of disassembly, checking for geometric accessibility for removal of parts. Since Archimedes plans only for straight-line motions, and this assembly contained a number of flexible parts, such as cables, that could not use straight-line assembly motions, the part-mating operations involving those parts were overrode. After loading and faceting all the parts, Archimedes took approximately three minutes to find all contacts and produce a disassembly plan. 
Intentionally Left Blank. 


\section{Automating Cost Estimates for Life Cycle Assembly Processes}

When a product concept emerges, the manufacturing engineer is asked to sketch out a production strategy and estimate its cost. The engineer is given an initial product design, along with a schedule of expected production volumes. The engineer then determines the best approach to manufacturing the product, comparing a variety of alternative production strategies. The engineer must consider capital cost, operating cost, lead-time, and other issues in an attempt to maximize profits. After making these basic choices and sketching the design of overall production, the engineer produces estimates of the required capital, operating costs, and production capacity. This process may iterate as the product design is refined in order to improve its performance or manufacturability.

The focus of this chapter is on the development of computer tools to aid manufacturing engineers in their decision-making processes. This computer software tool provides a framework in which accurate cost assembly and disassembly estimates can be seamlessly derived from design requirements at the start of any engineering project. The result is faster cycle times through firstpass success; lower life cycle cost due to requirements-driven design and accurate cost estimates derived early in the process. 


\subsection{Introduction}

Success in today's market demands rapid product development to meet customer expectations at a fair price. To do this, most companies must rethink their business strategies and revamp their product development environments. Products must be designed by integrated teams who work together to create new ways of doing business in order to produce better products faster and more efficiently. In order to achieve this goal, the integrated product development team needs information and tools that will facilitate flexibility, innovation, speed, accuracy, and process improvement. Computer-aided assembly planning software tools with cost-estimate capabilities will help achieve this goal.

A framework for calculating cost estimates has been integrated into the Archimedes $3.0^{\odot}$ System. The system calculates cost, in dollars or other units, associated with the assembly or disassembly of a product. This is an extremely important feature in that it provides a powerful tool for comparing costs of competing designs, upgrade vs. new product, etc.

This chapter describes the principles and implementation of a framework that supports an interactive system to plan, optimize, simulate, visualize, and document assembly and disassembly processes. The chapter further describes an integrated tool, the Design-for-Life-Cycle Cost Module, in which accurate cost estimates can be seamlessly derived from design requirements at the start of any engineering project. The result is faster cycle times through first-pass success; lower life cycle cost due to requirements-driven design and accurate cost estimates derived early in the process. In this work, emphasis has been placed on automatic and computer-aided generation, optimization, simulation, documentation, and "design-for" feedback of life cycle assembly plans derived from product CAD data. In Section 2 the cost module is introduced while Section 3 describes the implementation of the cost module. Section 4 describes experiments using the cost tool.

\subsection{Integrated Design-to-Cost}

Software has been added to the Archimedes $3.0^{\odot}$ System that allows the user to easily estimate the cost and time associated with the assembly of a product. Initial cost estimates are based on generic "handling" costs (e.g., the cost of an "insertion") and on the item (part) costs. As more information becomes available, the user may refine these costs and times by manually editing generic cost files. After Archimedes $3.0^{\odot}$ has generated an assembly plan, cost and time estimates are calculated and presented to the user in the form of a spreadsheet. Costs for specific assembly steps may be edited to further refine cost estimates.

\subsection{Interactivity and Implementation}

The user interface is critical to effectiveness and user acceptance of an interactive planing system. Chapter 2, Section 2 gives an overview of the Archimedes $3.0^{\odot}$ user interface. 
After Archimedes $3.0^{\circ}$ has generated an assembly plan, cost and time estimates may be obtained by selecting the Design-for-Lifecycle-Cost Analysis (DFLC Analysis) option from the File menu on the main Archimedes $3.0^{\odot}$ panel. The DFLC analysis module generates an initial assembly process description. This is represented in a tabulated window (similar to a spreadsheet) as shown in Figure 6.1. Each row of the table corresponds to a different process step while the columns of the table describe different aspects of a step. The table initially describes a series of part (or subassembly) placement or removal operations listed in the order that the movements were generated by the Archimedes $3.0^{\odot}$ assembly planner algorithms.

Upon startup, the DFLC analysis software checks for three files containing cost and time information. The "specific" cost and time file contains information that is specific to these assembly operations. The "generic" handling cost file contains information that may be applied to assembly operations of this type. The item cost file contains cost information for each item or part. None of these files are required for use of the DFLC Analysis. However their existence, especially that of the generic handling cost file and item cost file, significantly simplify analysis for the user. The user may generate the generic handling cost and item cost files using a simple text editor or other costing software that contains the appropriate information. The specific cost file is usually generated within Archimedes 3.0 $0^{\odot}$.

A generic handling cost file consists of multiple instances of the following information. These files have names of the form "asm.gcst" where "asm" is the name of the assembly.

Generic action (e.g., INSERT, INSERT WELD, or REORIENT -- Archimedes $3.0^{\odot}$ defined)

Comment (e.g., "Simple Insert" or "Insert with weld" -- user defined)

Handling_Cost (e.g., 1 -- user defined)

An item cost file consists of multiple instances of the following information and have a "icst" filename extension.

Item name (e.g., 399426-u-a or ms51957-1; this name is based on the item's CAD name)

Item cost (e.g., 1.23 -- user defined)

A specific cost and time file consists of multiple instances of the information listed below and have a "scst" filename extension.

Specific action (e.g., INSERT THREAD2: ms51957-1_15 with sh-r42886-000-u_08

-- Archimedes $3.0^{\oplus}$ defined)

Comment (e.g., "Delicate insertion" -- user defined)

Handling_Cost (e.g., 1.00 -- user defined)

Material_Cost (e.g., 2 -- user defined)

Step_Time (e.g., 0.1 -- user defined) 
Changes in entries for specific actions are usually made via the spreadsheet discussed below.

The DFLC analysis software assigns costs and times based on the information found in the cost files discussed above. First it looks in the specific-cost file for information. If no information is available for a particular assembly step, it estimates the handling cost based on information in the generic handling cost file, estimates the material cost based on information in the item cost file, and sets the time for that assembly step to zero.

After the initial costing is completed using the file information, a window opens and produces a table showing the individual assembly operations and process steps, handling cost, material cost, total cost, step time, and total time.

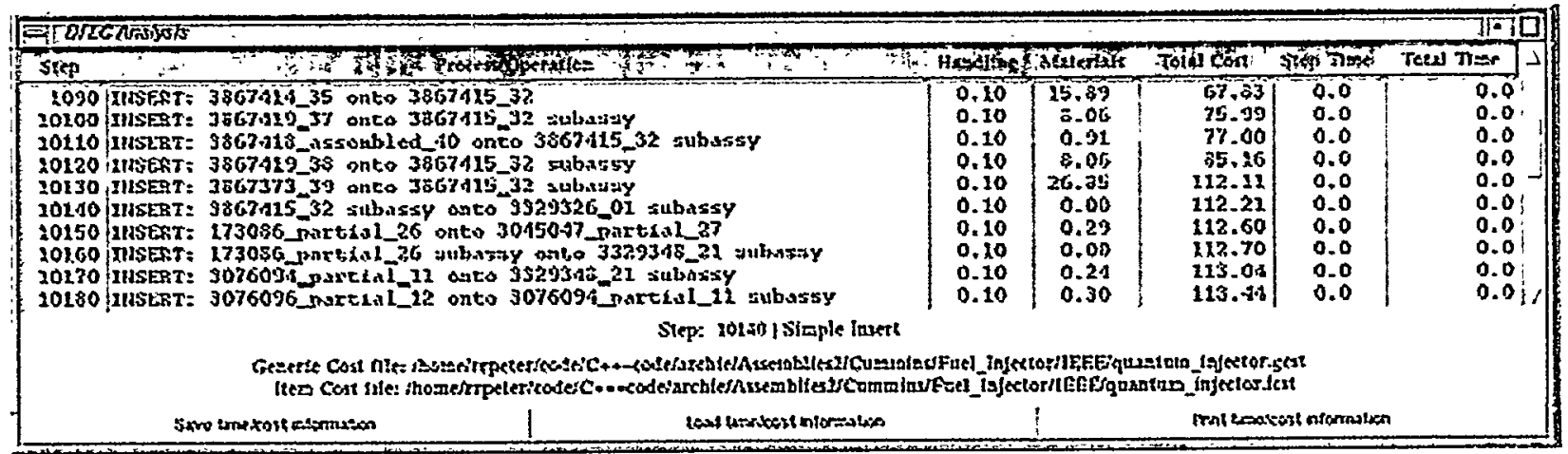

Figure 6.1. Archimedes $3.0^{\oplus}$ generated cost analysis spreadsheet.

Associated with the spreadsheet is the graphics visualization window. A double-left click on the Step field moves the assembly animation to the start of that operation. Animation window controls can be used to view that particular operation. Figures 6.1 and 6.2 demonstrate this concept. The process step associated with Step 10140 in the spreadsheet (Figure 6.1) is shown in the graphics animation window below.

A double-left click on the Process/Operation field allows one to edit a comment attached to that particular operation. The comment associated with a particular assembly step is shown near the bottom of the window. One can edit the handling cost, material cost, and step time fields directly by double-left clicking on them. The total cost and total time fields are automatically updated.

Below the cost/time table is the comment for the selected assembly step. Immediately below the comment is a listing of the files used for costing the assembly; zero to three files may be listed. The information shown in Figure 6.1 is estimating costs and times using only a generic handling cost file and an item cost file. 


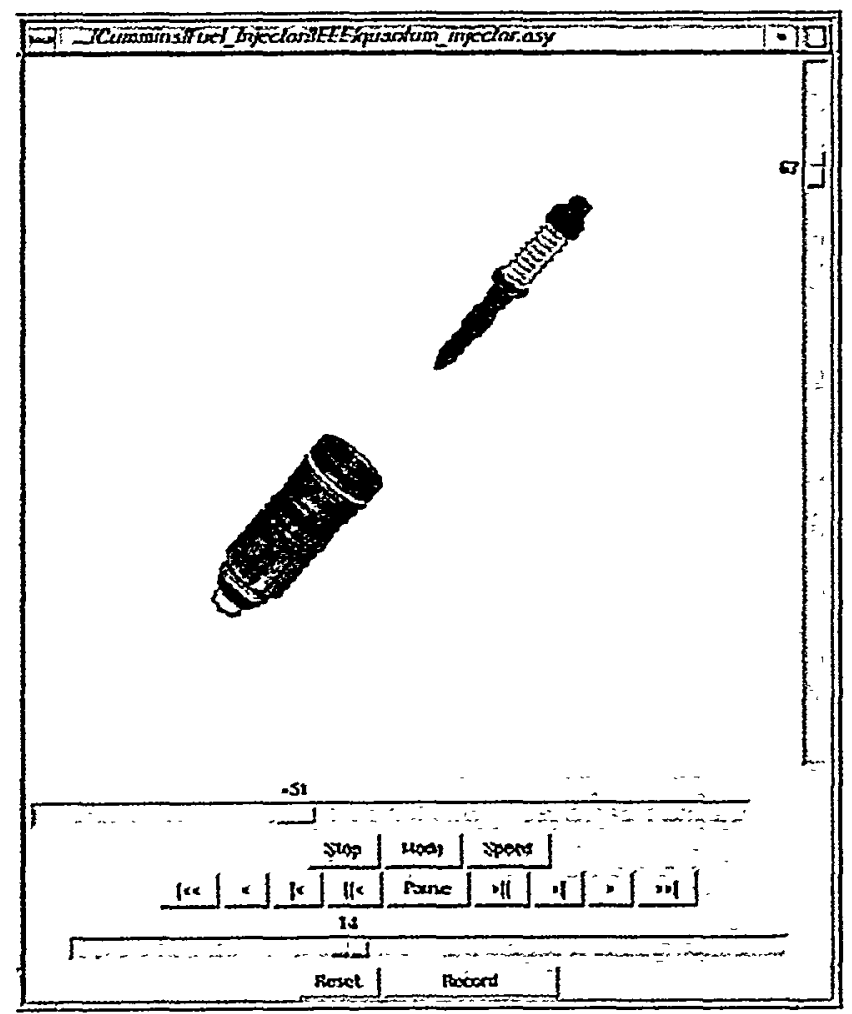

Figure 6.2. Archimedes $3.0^{\odot}$ frame-grab to associate with Step 1080 in Figure 6.1.

The three bottom buttons of the table (see Figure 6.1) allow input/output operations. Information contained in the table can be saved for later use with the "Save time/cost information" button; its output is a "specific cost and time file" which was previously discussed. The "Load time/cost information" allows one to use another file for specific cost and time information and overwrites what is currently in the table. Finally, the "Print time/cost information" outputs the table data to a file, which can be printed for later use.

\subsection{Experimentation}

The framework for automating cost estimates for life cycle assembly processes has been briefly described. However, due to the newness of the integrated cost module, application and testing of the cost module itself has been limited. The Fuel Systems Department at Cummins Engine Company in Columbus, Indiana has been very instrumental in the development of the cost module and its integration with the Archimedes $3.0^{\odot}$ planner. Cummins has assisted the development by providing suggestions of display formats and by providing real data for testing nurnoses Cirmmins also received a test-and-evaluation conv of the software to help guide future 
Intentionally Left Blank. 


\section{Small Smart Machines - A Framework for Automating Design}

Although somewhat disparate from the work described in the previous chapters on computeraided life cycle assembly analysis, this chapter discusses the development and implementation of a computerized framework used to automate the design processes and automatically generate cost estimates for the manufacture of small vehicular robots, or small smart machines (SSMs). However, theoretically, constraints truly are the underlying principles. The system is written in $\mathrm{CH}$ and implemented in Tcl/Tk. The computer algorithms are designed to read in customerspecified mission requirements and generate a set of Sandia design requirements using a components database designed and developed using pre-existing SSMs at Sandia. The system then couples the datum to produce a cost-effective machine design based on a user-defined optimization criteria. Mission requirements include the following: type, payload, computational, operating environment, travel range, communication range, duty cycle, duration, dimensional footprint, quantity, budget, schedule, and optimization criteria. The Sandia design requirements include the following: chassis, motors, batteries, cameras, sensors, radios, pivot bearings, CPUs, and actuators. Optimization rules and calculations that are implemented include cost of the machine, weight of the machine, size in volume, and power consumption. 


\title{
7.1 Motivation
}

The motivation for developing the tool was the need to accurately and quickly estimate costs, including design, test, production, and (perhaps later) training, operation and maintenance, of small vehicular robots or small smart machines (SSMs) for various scenarios. The tool needed to be able to estimate costs robots ranging from one to a million SSMs, and provide decision support for tradeoffs between mother/child robots.

\subsection{Implementation}

The system is written in $\mathrm{C}++$ and implements in Tcl/Tk. The computer algorithms are designed to read in customer-specified mission requirements and generate a set of Sandia design requirements using the components database described below and then couple the datum to produce a cost-effective machine design based on a user-defined optimization criteria. Optimization rules and calculations that are implemented include cost of the machine, weight of the machine, size in volume, and power consumption. The program runs on the UNIX platform.

\subsection{System Inputs}

There are two modes of input: interactive and ascii. The interactive input options are specified in Table. 7.1. The options include mission requirements and design requirements. Mission requirements include the following: type, payload, computational, operating environment, travel range, communication range, duty cycle, duration, dimensional footprint, quantity, budget, schedule, and optimization criteria. The Sandia design requirements include the following: chassis, motors, batteries, cameras, sensors, radios, pivot bearings, CPUs, and actuators.

The database is written in text and includes information:

Mission Requirements

SNL Design Requirements

Components Data

The sub-directory format is shown below:

\author{
$\sim /$ ssmcost \\ $\sim /$ ssmcost/Input \\ / /ssmcost/Input/Mission_Requirements \\ ／ssmcost/Input/SNL_Design_Requirements \\ r/ssmcost/Input/Components_Data
}




\subsection{Optimization Criteria}

Optimization rules that are currently implemented in the system are:

Cost: total cost of $n$ machines is the sum of the design times plus the installation/assembly times all times the labor rates

Weight: total weight of a machine is the sum of all component weights

Size: total size of a machine is the sum of the $\mathrm{L}^{*} \mathrm{~W}^{*} \mathrm{H}$ of all its components

Power: total power required for a machine is the of the sum of all component power consumption

\subsection{User Interface}

Shown below in Figure 7.1 is the graphical-user interface for the cost tool. In a typical session, the customer specifies their needs by clicking on the various options displayed in the left most column.

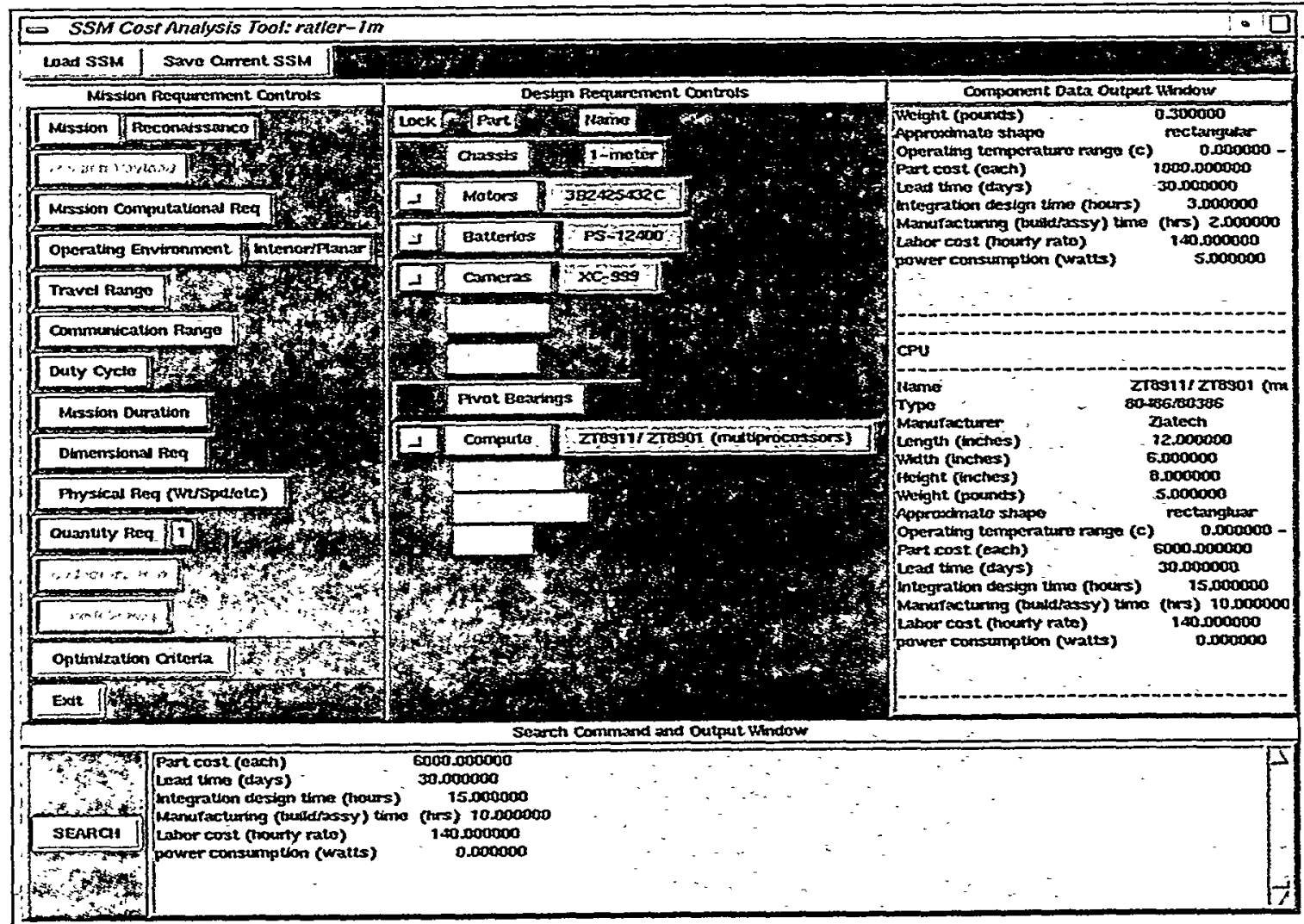

Figure 7.1: Small Smart Machine design system. 
The user then clicks on the "SEARCH" button located in the lower left-hand corner. The search algorithm implemented is an exhaustive search of the SNL database, using the optimization criteria to generate a feasible robot design. The optimal design parameters are displayed in the top-middle window, while the costs are displayed in the bottom window. The window on the right displays the parameters of the components. The user is allowed to modify the design by selecting and locking specific components using the buttons in the design requirements column. The user then SEARCH button to see the effect of the design modification. 


\section{Distribution:}

\begin{tabular}{|c|c|c|}
\hline MS & 0188 & LDRD Office, 4001 \\
\hline 1 & 0507 & C. M. Hart, 2600 \\
\hline 1 & 1002 & P. J. Eicker, 15200 \\
\hline 1 & 1005 & R. W. Shaum, 15201 \\
\hline 1 & 1004 & R. W. Harrigan, 15221 \\
\hline 1 & 1010 & M. E. Olson, 15222 \\
\hline 1 & 1006 & P. Garcia, 15271 \\
\hline 20 & 1008 & T. L. Calton, 15221 \\
\hline 10 & 1008 & S. Blauwkamp, 15200 Library, 15221 \\
\hline 1 & 9018 & Central Technical Files, $8940-2$ \\
\hline 2 & 0899 & Technical Library, 4916 \\
\hline & 0612 & Review \& Approval Desk, 4912 \\
\hline & & For DOE/OSTI \\
\hline & 0161 & Patent and Licensing Office, 11500 \\
\hline
\end{tabular}

